\title{
HOMOGENIZATION OF THE DIRICHLET PROBLEM FOR A SYSTEM OF QUASILINEAR ELLIPTIC EQUATIONS IN A DOMAIN WITH FINE-GRAINED BOUNDARY
}

\author{
Mamadou SANGO \\ Department of Mathematics, Vista University, Private Bag X1311, Silverton 0127, Pretoria, South Africa \\ Received 14 December 1999, revised 22 February 2001
}

ABSTRACT. - We study the Dirichlet problem for a system of nonlinear elliptic equations of Leray-Lions type in a sequence of domains $\Omega^{(s)}, s=1,2, \ldots$, with fine-grained boundaries. Under appropriate structure conditions on the system and the geometry of $\Omega^{(s)}$, we prove that the sequence of solutions of the problem converges in suitable topologies to the solution of a limit problem which contains an additional term of capacity type. We construct the limit problem.

(C) 2003 L'Association Publications de l'Institut Henri Poincaré. Published by Elsevier B.V. All rights reserved

RÉSUMÉ. - Nous étudions le problème de Dirichlet pour un système d'équations non linéaires élliptiques de type Leray-Lions dans une suite de domaines $\Omega^{(s)}, s=1,2, \ldots$, avec des frontières finement granulées. Sous des conditions de structure appropriées que nous imposons sur le système et sur la géometrie de $\Omega^{(s)}$, nous démontrons que la suite de solutions du problème tend dans des topologies appropriées vers la solution d'un problème limite qui contient un terme additionnel de type capacitaire. Nous construisons le problème limite.

(C) 2003 L'Association Publications de l'Institut Henri Poincaré. Published by Elsevier B.V. All rights reserved

\section{Introduction}

Let $\Omega$ be a bounded domain in $\mathbf{R}^{n}$ and for each given positive integer $s$ let there be defined a system $\left\{F_{i}^{(s)} ; i=1, \ldots, I(s)\right\}$ of closed, pairwise disjoint sets lying inside $\Omega$. In the domain $\Omega^{(s)}=\Omega \backslash \bigcup_{i=1}^{I(s)} F_{i}^{(s)}$, we consider the boundary value problem for a system of nonlinear elliptic equations

$$
\begin{gathered}
\sum_{l=1}^{n} \frac{\partial}{\partial x_{l}} A_{l}^{j}\left(x, \frac{\partial u}{\partial x}\right)=0 \quad \text { in } \Omega^{(s)} ; j=1, \ldots, N, \\
u(x)=f(x) \quad \text { on } \partial \Omega^{(s)},
\end{gathered}
$$

where $f(x)=\left(f_{1}(x), \ldots, f_{N}(x)\right)^{T}$ is a given vector-function in $\bar{\Omega}, u(x)=\left(u_{1}(x), \ldots\right.$, $\left.u_{N}(x)\right)^{T}, \quad \frac{\partial u}{\partial x}=\left(\frac{\partial u_{j}}{\partial x_{l}}\right)_{j=1, \ldots, N ; l=1, \ldots, n}$ is the gradient of $u, A^{j}=A^{j}(x, p)=$ 
$\left(A_{l}^{i}(x, p)\right)_{l=1, \ldots, n}$ is a vector function from $\Omega \times \mathbf{R}^{N n}$ into $\mathbf{R}^{n}$ with $p=\left(p_{l}^{i}\right)_{i=1, \ldots, N ; l=1, \ldots, n}$ $\in \mathbf{R}^{N n}, \partial \cdot$ denotes the boundary of a set $\cdot$. We shall assume throughout that $A_{l}^{i}=A_{l}^{i}(x, p)$ has the representation

$$
A_{l}^{j}(x, p)=\sum_{i=1}^{N} a_{l i}(x, p) p_{i}^{j} \quad \text { for } p \in \mathbf{R}^{N n}
$$

For an open set $\cdot$, we shall denote by $W_{m}^{1}(\cdot, N)$ the direct product of $N$ copies of the Sobolev space $W_{m}^{1}(\cdot)$ and by $\stackrel{\circ}{m}_{m}^{1}(\cdot, N)$ the set of vector-functions from $W_{m}^{1}(\cdot, N)$ which vanish on $\partial \cdot$. For a vector $v \in \mathbf{R}^{M}$ with components $v_{i}(i=1, \ldots, M)$, we denote its Euclidean norm by $|v|$, i.e., $|v|=\left(\sum_{i=1}^{M} v_{i}^{2}\right)^{1 / 2}$.

In this work we investigate the possibilities of approximating the problem (1)-(2) in the perforated domain $\Omega^{(s)}$ by a new homogenized problem in $\Omega$ whose solution is the limit of the sequence $u_{s}$ of solutions of (1)-(2) as $s \rightarrow \infty$. In the scalar case, i.e., when $N=1$, this problem has been studied by Skrypnik under various conditions on the geometry of $\Omega^{(s)}$ in many papers among which we cite [16-18] (the geometric restrictions in this paper are the weakest among those considered by the author so far). These works represent the nonlinear version of the theory invented by Marchenko and Khruslov (see [10]) for the study of linear elliptic boundary value problems in domains with fine-grained boundaries; the limit problem in this theory contains an additional term involving a function of capacity type. We note that a different approach has been elaborated by Cioranescu and Murat [3] and further developed in many other papers (see for instance [2,5-7], and the references therein); it does not require any conditions on the perforation and the limit problem contains an additional term involving a Borel measure which may assume infinite values. At the present moment many powerful methods in homogenization have been developed and an abundant literature on various aspects of the theory exist in form of monographs $[1,4,10,17,12,19]$, just to cite a few.

Some considerable difficulties arise at key turning points of our work, due to the nature of the system of nonlinear equations that we are dealing with, in particular in the appropriate choice of some auxiliary model problems whose solutions (satisfying some special boundary conditions) and their a priori estimates are of paramount importance in the asymptotic analysis of problem (1)-(2); they are needed for the construction of the asymptotic expansion of the solutions of (1)-(2) and the test function used for the derivation of the limit problem. The results of this work have been announced in [14].

The work is organized as follows. In Section 2, we formulate the conditions ensuring that any solution $u_{s}$ of problem (1)-(2) exists for each $s$ and the sequence is bounded in $W_{m}^{1}\left(\Omega^{(s)}, N\right)$ (in particular the system has a Leray-Lions structure), using Moser's iteration technics we prove that it is uniformly bounded. For a background on Moser's method, we refer to his celebrated paper [11] and the work of Serrin [15]. Next we introduce an auxiliary model problem which plays a crucial role in the sequel and the geometric conditions on $\Omega^{(s)}$ under which we investigate (1)-(2), we formulate our main result. In Section 3, we derive some sharp pointwise and integral estimates for solutions of the model problem, some of which have been obtained by us in [13]. In Section 4, we construct an appropriate asymptotic expansion with a remainder term for the sequence 
of solutions $u_{s}$ of problem (1)-(2), we prove that the sequence converges strongly in $W_{p}^{1}(\Omega, N)$ for $p \in(1, m)$, and that the remainder term converges strongly to zero in $W_{m}^{1}(\Omega, N)$. In Section 5, we construct the problem of which the limit $u_{0}$ of $u_{s}$ is a solution.

\section{Hypotheses and formulation of main results}

1) We assume that the functions $A_{l}^{j}$ are Caratheodory functions in $\bar{\Omega}$, i.e., they are defined for all $x \in \bar{\Omega}$ and $p \in \mathbf{R}^{N n}$, continuous in $p$ for a.e. $x \in \bar{\Omega}$ and measurable in $x$ for any $p \in \mathbf{R}^{N n}$.

2) The functions $A_{l}^{j}$ satisfy the following structure conditions: there exist the constants $v_{1}, v_{2}, v_{3}$ and $m$, with $2 \leqslant m<n$ such that for every vector $\xi=\left(\xi_{1}, \ldots, \xi_{n}\right)$

$$
\sum_{i, l=1}^{n} a_{l i}(x, p) \xi_{l} \xi_{i} \geqslant v_{1}|p|^{m-2}|\xi|^{2},
$$

and for every $p, q \in \mathbf{R}^{N n} ; p=\left(p^{j}\right)_{j=1, \ldots, N}, q=\left(q^{j}\right)_{j=1, \ldots, N}$ we have

$$
\begin{gathered}
\sum_{j=1}^{N}\left(p^{j}-q^{j}\right)\left(A^{j}(x, p)-A^{j}(x, q)\right) \geqslant v_{2}|p-q|^{m}, \\
\left|A^{j}(x, p)-A^{j}(x, q)\right| \leqslant\left[1+v_{3}\left(|p|^{m-2}+|q|^{m-2}\right)\right]|p-q|, \quad j=1, \ldots, N .
\end{gathered}
$$

We shall often use the symbol $C$ for nonessential constants depending on the data.

We shall call the vector function $u \in W_{m}^{1}\left(\Omega^{(s)}, N\right)$ a weak solution of problem (1)-(2) if, $u \in f+\stackrel{\circ}{W_{m}^{1}}\left(\Omega^{(s)}, N\right)$ with $f$ being a function which belongs to $W_{m}^{1}(\Omega, N)$, and for any vector function $\varphi(x)=\left(\varphi_{1}(x), \ldots, \varphi_{N}(x)\right) \in \stackrel{\circ}{W_{m}^{1}}\left(\Omega^{(s)}, N\right)$, the integral identity

$$
\sum_{j=1}^{N} \sum_{l=1}^{n} \int_{\Omega^{(s)}} A_{l}^{j}\left(x, \frac{\partial u}{\partial x}\right) \frac{\partial \varphi_{j}}{\partial x_{l}} d x=0
$$

holds.

From the results of Leray and Lions [9], we have

THEOREM 1.- Under the conditions 1$)$ and 2$)$, if $f \in W_{m}^{1}(\Omega, N)$, then the problem (1)-(2) has at least one solution $u_{s} \in f+\stackrel{\circ}{W_{m}^{1}}\left(\Omega^{(s)}, N\right)$ and there exists a positive constant $M_{1}$ independent of $s$, such that for all $s$, the inequality

$$
\left\|u_{s}\right\|_{W_{m}^{1}\left(\Omega^{(s)}, N\right)} \leqslant M_{1}
$$

holds.

Let $u_{s}$ be one of the solution of problem (1)-(2) satisfying inequality (7). We extend $u_{s}$ to $\Omega$ by setting $u_{s}(x)=f(x)$ in $\Omega \backslash \Omega^{(s)}$. The resulting function that we denote without 
fear of confusion by $u_{s}$ belongs to $W_{m}^{1}(\Omega, N)$ and satisfies the inequality

$$
\left\|u_{s}\right\|_{W_{m}^{1}(\Omega, N)} \leqslant M_{1}+\|f\|_{W_{m}^{1}(\Omega, N)} .
$$

From this inequality and the weak compactness of bounded sets in $W_{m}^{1}(\Omega, N)$, we may assume, by passing if necessary to a subsequence, that $u_{s}$ converges weakly to a function $u_{0}$ in $W_{m}^{1}(\Omega, N)$.

In our investigations the boundedness of the sequence $u_{s}$ will be needed. We show that in the following

THEOREM 2. - Let the conditions 1) and 2) be satisfied and let $f$ be a function belonging to $W_{q}^{1}(\Omega, N)$ with $q>n$. Then there exists a constant $M$ independent of $s$ such that $u_{s}$ satisfies the estimate,

$$
\operatorname{vrai} \max _{x \in \Omega}\left|u_{s}(x)\right| \leqslant M \text {. }
$$

Before proving the theorem, we note that the membership of $f$ to $W_{q}^{1}(\Omega, N)$ with $q>n$ and the boundedness of $\Omega$ imply that $f$ is continuous in $\bar{\Omega}$ and

$$
\sup _{x \in \Omega}|f(x)| \leqslant C(\text { meas } \Omega)^{1 / n-1 / q}\left\|\frac{\partial f}{\partial x}\right\|_{L_{q}(\Omega)},
$$

with the constant $C$ depending only on $n, N$ and $q$.

Proof. - We use a modification of Moser's method. Let $w_{s}(x)=\left|u_{s}(x)-f(x)\right|$. It is clear that $w_{s} \in \stackrel{\circ}{W}_{m}^{1}\left(\Omega^{(s)}\right)$. We consider the positive real numbers $r, k$ and $K$ such that $r \geqslant 2,0<k<K$. We define the truncated function $\zeta: \mathbf{R}^{n} \ni x \mapsto \zeta(x) \in \mathbf{R}$ as

$$
\zeta(x)= \begin{cases}k & \text { if } 0 \leqslant|x| \leqslant k \\ |x| & \text { if } k<|x|<K \\ K & \text { if } K \leqslant|x|\end{cases}
$$

and set

$$
w_{s k}^{(K)}(x)=\zeta\left(u_{s}(x)-f(x)\right) .
$$

The function $w_{s k}^{(K)}$ so defined is clearly bounded and since $\zeta$ is Lipshitz, standard arguments as in the scalar case (see, e.g., [8, Chapter 2, § 3]) show that $w_{s k}^{(K)} \in W_{m}^{1}(\Omega)$. Let us denote by $\chi_{k}^{(K)}$ the characteristic function of the set $\left\{x \in \Omega^{(s)}: k<\left|u_{s}-f\right|<K\right\}$. We consider the function $\varphi(x)=\left(\varphi_{1}(x), \ldots, \varphi_{N}(x)\right)$, with

$$
\varphi_{j}(x)=\left(u_{s j}(x)-f_{j}(x)\right)\left[w_{s k}^{(K)}(x)\right]^{r}, \quad j=1, \ldots, N .
$$

Owing to the properties of $w_{s k}^{(K)}$ we see that $\varphi \in \stackrel{\circ}{W_{m}^{1}}\left(\Omega^{(s)}, N\right)$. Thus $\varphi$ is an admissible test function in the integral identity (6). We have

$$
\frac{\partial \varphi_{j}}{\partial x_{l}}=\frac{\partial\left(u_{s j}-f_{j}\right)}{\partial x_{l}}\left[w_{s k}^{(K)}\right]^{r}+r \sum_{i=1}^{N}\left(u_{s j}-f_{j}\right)\left(u_{s i}-f_{i}\right)\left[w_{s k}^{(K)}\right]^{r-2} \frac{\partial\left(u_{s i}-f_{i}\right)}{\partial x_{l}} \chi_{k}^{(K)} .
$$


Let us write the identity (6) in the form

$$
\begin{aligned}
& \sum_{j=1}^{N} \sum_{l=1}^{n} \int_{\Omega^{(s)}} A_{l}^{i}\left(x, \frac{\partial\left(u_{s}-f\right)}{\partial x}\right) \frac{\partial \varphi_{j}}{\partial x_{l}} d x \\
& \quad=\sum_{j=1}^{N} \sum_{l=1}^{n} \int_{\Omega^{(s)}}\left[A_{l}^{i}\left(x, \frac{\partial\left(u_{s}-f\right)}{\partial x}\right)-A_{l}^{i}\left(x, \frac{\partial u_{s}}{\partial x}\right)\right] \frac{\partial \varphi_{j}}{\partial x_{l}} d x .
\end{aligned}
$$

Substituting formula (10) in (11) and using the condition 2), we get

$$
\begin{aligned}
& \int_{\Omega^{(s)}}\left|\frac{\partial\left(u_{s}-f\right)}{\partial x}\right|^{m}\left[w_{s k}^{(K)}\right]^{r}(x) d x+r \int_{\Omega^{(s)}}\left|\frac{\partial\left(u_{s}-f\right)}{\partial x}\right|^{m-2}\left|\frac{\partial w_{s k}^{(K)}}{\partial x}\right|^{2}\left[w_{s k}^{(K)}\right]^{r} \chi_{k}^{(K)} d x \\
& \quad \leqslant C\left\{H_{1}+H_{2}+H_{3}\right\},
\end{aligned}
$$

where

$$
\begin{gathered}
H_{1}=(r+1) \int_{\Omega^{(s)}}\left|\frac{\partial f}{\partial x}\right|\left|\frac{\partial\left(u_{s}-f\right)}{\partial x}\right|\left[w_{s k}^{(K)}\right]^{r} d x, \\
H_{2}=(r+1) \int_{\Omega^{(s)}}\left|\frac{\partial f}{\partial x}\right|\left|\frac{\partial\left(u_{s}-f\right)}{\partial x}\right|^{m-1}\left[w_{s k}^{(K)}\right]^{r} d x, \\
H_{3}=(r+1) \int_{\Omega^{(s)}}\left|\frac{\partial f}{\partial x}\right|\left|\frac{\partial u_{s}}{\partial x}\right|^{m-2}\left|\frac{\partial\left(u_{s}-f\right)}{\partial x}\right|\left[w_{s k}^{(K)}\right]^{r} d x .
\end{gathered}
$$

By Young's inequality, we have

$$
\begin{gathered}
H_{1} \leqslant C_{\varepsilon_{1}}(r+1)^{\frac{m}{m-1}} \int_{\Omega^{(s)}}\left|\frac{\partial f}{\partial x}\right|^{\frac{m}{m-1}}\left[w_{s k}^{(K)}\right]^{r} d x+\varepsilon_{1} \int_{\Omega^{(s)}}\left|\frac{\partial\left(u_{s}-f\right)}{\partial x}\right|^{m}\left[w_{s k}^{(K)}\right]^{r} d x, \\
H_{2} \leqslant C_{\varepsilon_{2}}(r+1)^{m} \int_{\Omega^{(s)}}\left|\frac{\partial f}{\partial x}\right|^{m}\left[w_{s k}^{(K)}\right]^{r} d x+\varepsilon_{2} \int_{\Omega^{(s)}}\left|\frac{\partial\left(u_{s}-f\right)}{\partial x}\right|^{m}\left[w_{s k}^{(K)}\right]^{r} d x, \\
H_{3} \leqslant C_{\varepsilon_{3}}(r+1)^{m} \int_{\Omega^{(s)}}\left|\frac{\partial f}{\partial x}\right|^{m}\left[w_{s k}^{(K)}\right]^{r} d x+\varepsilon_{3} \int_{\Omega^{(s)}}\left|\frac{\partial\left(u_{s}-f\right)}{\partial x}\right|^{m}\left[w_{s k}^{(K)}\right]^{r} d x \\
+C(r+1)^{m} \int_{\Omega^{(s)}}\left|\frac{\partial f}{\partial x}\right|^{m-1}\left[w_{s k}^{(K)}\right]^{r} d x .
\end{gathered}
$$

Choosing $\varepsilon_{1}, \varepsilon_{2}$ and $\varepsilon_{3}$ sufficiently small in these inequalities, we get

$$
\begin{aligned}
& \int_{\Omega^{(s)}}\left|\frac{\partial\left(u_{s}-f\right)}{\partial x}\right|^{m}\left[w_{s k}^{(K)}\right]^{r} d x+r \int_{\Omega^{(s)}}\left|\frac{\partial\left(u_{s}-f\right)}{\partial x}\right|^{m-2}\left|\frac{\partial w_{s k}^{(K)}}{\partial x}\right|\left[w_{s k}^{(K)}\right]^{r} \chi_{k}^{(K)} d x \\
& \leqslant C(r+1)^{m}\left\{\int_{\Omega^{(s)}}\left|\frac{\partial f}{\partial x}\right|^{\frac{m}{m-1}}\left[w_{s k}^{(K)}\right]^{r} d x+\int_{\Omega^{(s)}}\left|\frac{\partial f}{\partial x}\right|^{m}\left[w_{s k}^{(K)}\right]^{r} d x\right.
\end{aligned}
$$




$$
\left.+\int_{\Omega^{(s)}}\left|\frac{\partial f}{\partial x}\right|^{m-1}\left[w_{s k}^{(K)}\right]^{r} d x\right\}
$$

Let us denote the integrals in the right-hand side of this inequality by $H_{11}, H_{12}$ and $H_{13}$ respectively. Using Young's and Hölder's inequalities, we estimate these integrals as follows.

$$
H_{11} \leqslant C\left\{\int_{\Omega^{(s)}}\left|\frac{\partial f}{\partial x}\right|^{q} d x\right\}^{\frac{m}{q(m-1)}}\left\{1+\int_{\Omega^{(s)}}\left[w_{s k}^{(K)}\right]^{(r+m) p_{1}}\right\}^{\frac{1}{p_{1}}}
$$

where $p_{1}=\frac{q(m-1)}{q(m-1)-m}$.

$$
H_{12} \leqslant C\left\{\int_{\Omega^{(s)}}\left|\frac{\partial f}{\partial x}\right|^{q} d x\right\}^{\frac{m}{q}}\left\{1+\int_{\Omega^{(s)}}\left[w_{s k}^{(K)}\right]^{(r+m) p_{2}}\right\}^{\frac{1}{p_{2}}},
$$

where $p_{2}=\frac{q}{q-m}$.

$$
H_{13} \leqslant C\left\{\int_{\Omega^{(s)}}\left|\frac{\partial f}{\partial x}\right|^{q} d x\right\}^{\frac{m-1}{q}}\left\{1+\int_{\Omega^{(s)}}\left[w_{s k}^{(K)}\right]^{(r+m) p_{3}} d x\right\}^{\frac{1}{p_{3}}},
$$

where $p_{3}=\frac{q}{q-m+1}$.

Let $Q=\max \left\{p_{1}, p_{2}, p_{3}\right\}$. Since $f \in W_{q}^{1}(\Omega, N)$, and

$$
\left|\frac{\partial w_{s k}^{(K)}}{\partial x}\right| \leqslant\left|\frac{\partial\left(u_{s}-f\right)\left(u_{s}-f\right)}{\partial x}\right|,
$$

it follows from the above estimations of $H_{1 k}, k=1,2,3$ and the inequality (12) that

$$
\int_{\Omega^{(s)}}\left|\frac{\partial w_{s k}^{(K)}}{\partial x}\right|^{m}\left[w_{s k}^{(K)}\right]^{r} d x \leqslant C(r+1)^{m}\left\{1+\int_{\Omega^{(s)}}\left[w_{s k}^{(K)}\right]^{(r+m) Q}(x) d x\right\}^{1 / Q} .
$$

Since $w_{s k}^{(K)}$ is bounded, we have that $\left[w_{s k}^{(K)}\right]^{t} \in W_{m}^{1}\left(\Omega^{(s)}\right)$ for any $t>1$. Thus (13) implies

$$
\int_{\Omega^{(s)}}\left|\frac{\partial\left[w_{s k}^{(K)}\right]^{1+(r / m)}}{\partial x}\right|^{m} d x \leqslant C(r+1)^{m}\left\{1+\int_{\Omega^{(s)}}\left[w_{s k}^{(K)}\right]^{(r+m) Q}(x) d x\right\}^{1 / Q} .
$$

The Sobolev embedding $W_{m}^{1}(\Omega) \hookrightarrow L_{\frac{n m}{n-m}}(\Omega)$ holds. Thus for any $r>0$, we have

$$
\int_{\Omega^{(s)}}\left[w_{s k}^{(K)}\right]^{r} d x \leqslant C\left\{\int_{\Omega^{(s)}}\left|\frac{\partial\left[w_{s k}^{(K)}\right]^{\frac{r(n-m)}{n m}}}{\partial x}\right|^{m} d x\right\}^{n /(n-m)} .
$$


By rescaling $r$ in (14), we obtain from (15) the inequality

$$
\int_{\Omega^{(s)}}\left[w_{s k}^{(K)}\right]^{r} d x \leqslant C(r+1)^{\frac{2 m n}{n-m}}\left\{1+\int_{\Omega^{(s)}}\left[w_{s k}^{(K)}\right]^{\frac{r(n-m)}{n} Q}(x) d x\right\}^{\frac{n}{Q(n-m)}} .
$$

Passing to the limit in this inequality as $k \rightarrow 0$ and $K \rightarrow \infty$, and applying Fatou's Lemma in the left-hand side and Lebesgue's dominated convergence theorem in the right-hand side, we get

$$
\int_{\Omega^{(s)}} w_{s}^{r} d x \leqslant C(r+1)^{\frac{2 m n}{n-m}}\left\{1+\int_{\Omega^{(s)}} w_{s}^{\frac{r(n-m)}{n} Q}(x) d x\right\}^{\frac{n}{Q(n-m)}} .
$$

Letting

$$
r=r_{i}=\frac{n m}{n-m}\left(\frac{n}{Q(n-m)}\right)^{i}, \quad i=0,1,2, \ldots
$$

and

$$
I_{i}=1+\int_{\Omega^{(s)}} w_{s}^{r_{i}}(x) d x,
$$

we obtain from (17) the recurrent inequality

$$
I_{i} \leqslant C\left[\left(\frac{n}{(n-m) Q}\right)^{\frac{2 n m}{n-m}}\right]^{i} I_{i-1}^{\sigma^{-1}},
$$

with $\sigma=\frac{Q(n-m)}{n}$. Iterating the inequality (18), we get

$$
I_{i}^{\sigma^{i}} \leqslant C^{\sigma^{i}+\sigma^{i-1}+\cdots+\sigma} A^{i \sigma^{i}+(i-1) \sigma^{i-1}+\cdots+\sigma} I_{0},
$$

where $A=\left[\frac{n}{Q(n-m)}\right]^{\frac{2 m n}{n-m}}$.

Let $U_{M}=\left\{x \in \Omega^{(s)}: w_{s}(x) \geqslant M\right\}$, with $M=\operatorname{vrai}_{\max } \operatorname{mo\Omega }_{x(s)} w_{s}(x)$ and assume that meas $U_{M}$, the Lebesgue measure of $U_{M}$ is different from zero. We have

$$
I_{i} \geqslant \int_{U_{M}} w_{s}^{r_{i}}(x) d x+1 \geqslant M^{\left(\frac{n m}{n-m}\right)^{\sigma^{i}}} \text { meas } U_{M} .
$$

Thus from (19), as $i$ approaches infinity, we get

$$
\text { vrai } \max _{x \in \Omega^{(s)}} w_{s}(x) \leqslant C\left\{1+\int_{\Omega^{(s)}} w_{s}^{\frac{n m}{n-m}}(x) d x\right\}^{\frac{n-m}{n m}} .
$$

This implies that

$$
\text { vrai } \max _{x \in \Omega^{(s)}}\left|u_{s}(x)\right| \leqslant C\left\{\left\|u_{s}-f\right\|_{L_{\frac{n m}{n-m}}(\Omega, N)}+\|f\|_{W_{q}^{1}(\Omega, N)}+1\right\} ;
$$


here we have used inequality (9). The theorem is proved.

We now state the conditions on the geometry of the set $\Omega^{(s)}$. Let $d_{i}^{(s)}$ be the diameter of the set $F_{i}^{(s)}$ and let $x_{i}^{(s)}$ be the center of the ball $B\left(x_{i}^{(s)}, d_{i}^{(s)}\right)$ of radius $d_{i}^{(s)}$ such that $F_{i}^{(s)} \subset \overline{B\left(x_{i}^{(s)}, d_{i}^{(s)}\right)}$. We denote by $r_{i}^{(s)}$ the distance from the ball $B\left(x_{i}^{(s)}, d_{i}^{(s)}\right)$ to the set $\bigcup_{j \neq i} B\left(x_{j}^{(s)}, d_{j}^{(s)}\right) \cup \partial \Omega$. We assume that the following conditions are satisfied: There exist the constants $C_{0}$ and $C_{1}$ independent of $i$ and $s$ such that

B1. $\lim _{s \rightarrow \infty} \max \left\{r_{i}^{(s)}\right\}=0, d_{i}^{(s)} \leqslant C_{1} r_{i}^{(s)}$.

B2.

$$
\sum_{i=1}^{I(s)}\left[\frac{\left[d_{i}^{(s)}\right]^{m(n-m)}}{\left[r_{i}^{(s)}\right]^{n}}\right]^{\frac{1}{m-1}} \leqslant C_{0} .
$$

For the formulation of another condition we introduce a model problem which will play a central role in our investigations. Let $\vec{k} \in \mathbf{R}^{N}$ and $k$ the euclidean norm of $\vec{k}$. We denote by $\vec{e}$ the unit $N$-dimensional vector $\frac{\vec{k}}{k}$, and set $\Omega_{i}^{(s)}=B\left(x_{i}^{(s)}, 1\right) \backslash F_{i}^{(s)}$. Let $\psi \in \mathbf{C}_{o}^{\infty}\left(B\left(x_{i}^{(s)}, 1\right)\right)$ with $\psi(x)=1$ in $B\left(x_{i}^{(s)}, \frac{1}{2}\right)$. For any $\vec{k}$, when $d_{i}<\frac{1}{2}$, we denote by $v_{i}^{(s)}(x, \vec{k})$, the vector-function from $k \psi\left(x-x_{i}^{(s)}\right) \vec{e}+\stackrel{\circ}{W}_{m}^{1}\left(\Omega_{i}^{(s)}, N\right)$ which satisfies the integral identity

$$
\sum_{j=1}^{N} \sum_{l=1}^{n} \int_{\Omega_{i}^{(s)}} A_{l}^{j}\left(x, \frac{\partial v_{i}^{(s)}}{\partial x}\right) \frac{\partial \varphi_{j}}{\partial x_{l}} d x=0,
$$

for any $\varphi(x)=\left(\varphi_{1}(x), \ldots, \varphi_{N}(x)\right) \in \stackrel{\circ}{W_{m}^{1}}\left(\Omega_{i}^{(s)}, N\right)$. Further the functions $v_{i}^{(s)}(x, \vec{k})$ are extended to $\Omega$ by setting $v_{i}^{(s)}(x, \vec{k})=k \psi\left(x-x_{i}^{(s)}\right) \vec{e}$ for $x \notin \Omega_{i}^{(s)}$. In other words $v_{i}^{(s)}(x, \vec{k})$ is a weak solution of the boundary value problem

$$
\begin{gathered}
\sum_{l=1}^{n} \frac{\partial}{\partial x_{l}} A_{l}^{j}\left(x, \frac{\partial v}{\partial x}\right)=0 \quad \text { in } \Omega_{i}^{(s)}, j=1, \ldots, N, \\
v(x)=k \psi\left(x-x_{i}^{(s)}\right) \vec{e} \quad \text { on } \partial \Omega_{i}^{(s)} .
\end{gathered}
$$

Under the conditions 1) and 2) the existence of a solution to the problem (21)-(22) follows from Leray and Lions [9]. We impose on the sets $F_{i}^{(s)}$ the following restriction:

C. Let $\vec{k}=\left(k_{1}, \ldots, k_{N}\right) \in \mathbf{R}^{N}$. For any $\alpha=1, \ldots, N$, there exists a continuous function $C^{(\alpha)}(x, \vec{k})$ such that for any subset $D \subset \Omega$, we have

$$
\begin{aligned}
& \int_{D} C^{(\alpha)}(x, \vec{k}) d x \\
& = \begin{cases}\lim _{s \rightarrow \infty} \sum_{i \in I_{s}(D)} \sum_{j=1}^{N} \frac{1}{k_{\alpha}} \int_{D} A^{j}\left(x, \frac{\partial v_{i}^{(s)}(x, \vec{k})}{\partial x}\right) \frac{\partial v_{i \alpha, j}^{(s)}(x, \vec{k})}{\partial x} d x & \text { if } k_{\alpha} \neq 0,\end{cases}
\end{aligned}
$$

where $v_{i \alpha, j}^{(s)}(x, \vec{k})(j=1, \ldots, N)$ are the components of the vector-function $v_{i \alpha}^{(s)}(x, \vec{k})$ which is a solution of problem (21)-(22) with $\vec{e}=\vec{e}^{(\alpha)} ; \vec{e}^{(\alpha)}$ being the $N$-dimensional 
vector whose $\alpha$ th component equals $k_{\alpha} /\left|k_{\alpha}\right|\left(k_{\alpha} \neq 0\right)$ and the remaining components equal zero, $I_{s}(D)=\left\{i=1, \ldots, I(s): x_{i}^{(s)} \in D\right\}$, and the convergence in the limit in (23) is uniform in $\vec{k}$ with $|\vec{k}|<\infty$. We can look at the vector-function $\vec{C}(x, \vec{k})=$ $\left(C^{(1)}(x, \vec{k}), \ldots, C^{(N)}(x, \vec{k})\right)$ as the vector-valued analog of the additional term of capacity type in the works [17] and [18].

Now we are in a position to formulate our main result.

THEOREM 3. - Let the conditions 1), 2), B1, B2 and C be satisfied. Assume that $f \in W_{q}^{1}(\Omega, N)$ with $q>n$, and let $u_{s}$ be the sequence of solutions of problem (1)(2) which satisfies inequality (7) and converges weakly to the vector-function $u_{0}$ in $W_{m}^{1}(\Omega, N)$. Then the sequence $u_{s}$ converges strongly to $u_{0}$ in $W_{p}^{1}(\Omega, N)$ for every $p \in(1, m)$, and the function $u_{0}$ is a solution to the boundary value problem

$$
\begin{gathered}
\sum_{l=1}^{n} \frac{\partial}{\partial x_{l}} A_{l}^{j}\left(x, \frac{\partial u}{\partial x}\right)+C^{(j)}(x, f(x)-u(x))=0 \quad \text { in } \Omega, j=1, \ldots, N, \\
u(x)=f(x) \quad \text { on } \partial \Omega .
\end{gathered}
$$

When $N=1$, this theorem is due to Skrypnik [17, Chap. 9]. The theorem will follow from a suitable asymptotic expansion of the solutions $u_{s}$ of problem (1)-(2) which plays an important role in the construction of the limit problem (24)-(25). The asymptotic expansion and the derivation of the limit problem rely on the solutions of the model problems (21)-(22) and some of their integral and pointwise a priori estimates. In the next section we deal with these a priori estimates.

\section{Integral and pointwise estimates of the solutions of problem (21)-(22)}

We start with

LEMMA 4. - Le the assumptions 1) and 2) be satisfied. Then for any vector $\vec{k} \neq 0$, any solution $v_{i}^{(s)}(x, \vec{k}) \in k \psi\left(x-x_{i}^{(s)}\right) \vec{e}+\stackrel{\circ}{W_{m}^{1}}\left(\Omega_{i}^{(s)}, N\right)$ of problem (21)-(22) satisfies the inequality

$$
\left|v_{i}^{(s)}(x, \vec{k})\right| \leqslant k
$$

Proof. - In the integral identity (20), let

$$
\varphi(x)= \begin{cases}0 & \text { if }\left|v_{i}^{(s)}(x, \vec{k})\right| \leqslant k \\ v_{i}^{(s)}(x, \vec{k})-k \vec{e} & \text { if }\left|v_{i}^{(s)}(x, \vec{k})\right|>k\end{cases}
$$

Let $E_{k}=\left\{x \in \Omega:\left|v_{i}^{(s)}(x, \vec{k})\right| \leqslant k\right\}$. Thus we have

$$
\int_{B\left(x_{i}^{(s)}, 1\right) \backslash E_{k}}\left|\frac{\partial v_{i}^{(s)}}{\partial x}\right|^{m} d x \leqslant \int_{B\left(x_{i}^{(s)}, 1\right) \backslash E_{k}} \sum_{j=1}^{N} A^{j}\left(x, \frac{\partial v_{i}^{(s)}}{\partial x}\right) \frac{\partial v_{i j}^{(s)}}{\partial x} d x=0 .
$$

This implies that either meas $B\left(x_{i}^{(s)}, 1\right) \backslash E_{k}=0$ or $v_{i}^{(s)}$ has constant components. In the last case $v_{i}^{(s)}(x, k)=k \vec{e}$. Hence in both situations we have (26). 
In analogy with [13], we have

THEOREM 5. - Let the conditions 1) and 2) be satisfied. Then there exist the constants $K_{1}, K_{2}$ and $K_{3}$ depending only on $N, n, m, v_{1}, v_{2}, v_{3}$ such that for $i=$ $1, \ldots, I(s), s=1,2, \ldots$, for any solution $v_{i}^{(s)}(x, \vec{k}) \in k \psi\left(x-x_{i}^{(s)}\right) \vec{e}+\stackrel{\circ}{W_{m}^{1}}\left(\Omega_{i}^{(s)}, N\right)$ of problem (21)-(22) the following claims hold:

1.

$$
\int_{B\left(x_{i}^{(s)}, 1\right)}\left|\frac{\partial v_{i}^{(s)}}{\partial x}\right|^{m} d x \leqslant K_{1} k^{m}\left[d_{i}^{(s)}\right]^{n-m} .
$$

2. For $0<t<k$ and $E_{t}=\left\{x \in B\left(x_{i}^{(s)}, 1\right):\left|v_{i}^{(s)}(x, \vec{k})\right| \leqslant t\right\}$

$$
\int_{E_{t}}\left|\frac{\partial v_{i}^{(s)}}{\partial x}\right|^{m} d x \leqslant K_{2} t k^{m-1}\left[d_{i}^{(s)}\right]^{n-m}
$$

3.

$$
\left|v_{i}^{(s)}(x, \vec{k})\right| \leqslant K_{3} k\left[\frac{d_{i}^{(s)}}{\left|x-x_{i}^{(s)}\right|}\right]^{\frac{n-m}{m-1}},
$$

for any $x \in B\left(x_{i}^{(s)}, 1\right) \backslash B\left(x_{i}^{(s)}, d_{i}^{(s)}\right)$.

We prove the following

THEOREM 6. - Let $\bar{k}=\left(\bar{k}_{1}, \ldots, \bar{k}_{N}\right)$ and $\hat{k}=\left(\hat{k}_{1}, \ldots, \hat{k}_{N}\right)$ be some $N$-dimensional vectors with $|\bar{k}|,|\hat{k}|<\infty$ and let $v_{i}^{(s)}(x, \bar{k}) \in \bar{k} \psi\left(x-x_{i}^{(s)}\right)+{\stackrel{\circ}{W_{m}}}^{1}\left(\Omega_{i}^{(s)}, N\right), v_{i}^{(s)}(x, \hat{k}) \in$ $\hat{k} \psi\left(x-x_{i}^{(s)}\right)+\stackrel{\circ}{W_{m}^{1}}\left(\Omega_{i}^{(s)}, N\right)$ be solutions of problem (21)-(22). If the conditions 1$)$ and 2) are satisfied, then the following inequalities hold:

$$
\int_{B\left(x_{i}^{(s)}, 1\right)}\left|\frac{\partial}{\partial x}\left[v_{i}^{(s)}(x, \bar{k})-v_{i}^{(s)}(x, \hat{k})\right]\right|^{m} \leqslant K_{4}|\bar{k}-\hat{k}|^{\frac{m}{m-1}}\left[d_{i}^{(s)}\right]^{n-m},
$$

and for $\bar{k}_{\alpha}, \hat{k}_{\alpha} \neq 0$,

$$
\begin{aligned}
& \mid \sum_{j=1}^{N} \int_{B\left(x_{i}^{(s)}, 1\right)}\left\{A^{j}\left(x, \frac{\partial v_{i}^{(s)}(x, \bar{k})}{\partial x}\right) \frac{1}{\bar{k}_{\alpha}} \frac{\partial v_{i \alpha}^{(s)}(x, \bar{k})}{\partial x}\right. \\
& \left.\quad-A^{j}\left(x, \frac{\partial v_{i}^{(s)}(x, \hat{k})}{\partial x}\right) \frac{1}{\hat{k}_{\alpha}} \frac{\partial v_{i \alpha}^{(s)}(x, \hat{k})}{\partial x}\right\} d x\left|\leqslant K_{5}\right| \bar{k}-\left.\hat{k}\right|^{\frac{1}{m-1}}\left[d_{i}^{(s)}\right]^{n-m} ;
\end{aligned}
$$

the constants $K_{4}$ and $K_{5}$ depend only on $N, n, m, v_{1}, v_{2}, v_{3}$. 
Proof. - For simplicity, we write $\bar{v}(x)=v_{i}^{(s)}(x, \bar{k}), \hat{v}(x)=v_{i}^{(s)}(x, \hat{k})$. Substituting the test function

$$
\varphi(x)=\bar{v}(x)-\hat{v}(x)-|\bar{k}-\hat{k}| \psi\left(\frac{x-x_{i}^{(s)}}{d_{i}^{(s)}}\right) \vec{q},
$$

with $\vec{q}=\frac{\bar{k}-\hat{k}}{|\bar{k}-\hat{k}|}, \psi \in \mathbf{C}_{o}^{\infty}\left(B\left(x_{i}^{(s)}, 1\right)\right), \psi(y)=1$ in $B\left(x_{i}^{(s)}, \frac{1}{2}\right)$, in the integral identity (20) for $\bar{v}$ and $\hat{v}$, and subtracting the resulting equations from each other, and using the condition 2), we get

$$
\int_{B\left(x_{i}^{(s)}, 1\right)}\left|\frac{\partial(\bar{v}-\hat{v})}{\partial x}\right|^{m} d x \leqslant C\left\{I_{1}+I_{2}+I_{3}\right\}
$$

where

$$
\begin{gathered}
I_{1}=\frac{|\bar{k}-\hat{k}|}{d_{i}^{(s)}} \int_{B\left(x_{i}^{(s)}, 1\right)}\left|\psi^{\prime}\right|\left|\frac{\partial(\bar{v}-\hat{v})}{\partial x}\right| d x, \\
I_{2}=\frac{|\bar{k}-\hat{k}|}{d_{i}^{(s)}} \int_{B\left(x_{i}^{(s)}, 1\right)}\left|\psi^{\prime}\right|\left|\frac{\partial \bar{v}}{\partial x}\right|^{m-2}\left|\frac{\partial(\bar{v}-\hat{v})}{\partial x}\right| d x, \\
I_{3}=\frac{|\bar{k}-\hat{k}|}{d_{i}^{(s)}} \int_{B\left(x_{i}^{(s)}, 1\right)}\left|\psi^{\prime}\right|\left|\frac{\partial \hat{v}}{\partial x}\right|^{m-2}\left|\frac{\partial(\bar{v}-\hat{v})}{\partial x}\right| d x .
\end{gathered}
$$

By Young's inequality, we have

$$
\begin{gathered}
I_{1} \leqslant \varepsilon \int_{B\left(x_{i}^{(s)}, 1\right)}\left|\frac{\partial(\bar{v}-\hat{v})}{\partial x}\right|^{m} d x+C_{\varepsilon}|\hat{k}-\bar{k}|^{\frac{m}{m-1}}\left[d_{i}^{(s)}\right]^{n-m} \\
I_{2} \leqslant \varepsilon \int_{B\left(x_{i}^{(s)}, 1\right)}\left|\frac{\partial(\bar{v}-\hat{v})}{\partial x}\right|^{m} d x+C_{\varepsilon}\left[\frac{|\bar{k}-\hat{k}|}{d_{i}^{(s)}}\right]^{\frac{m}{m-1}} \int_{B\left(x_{i}^{(s)}, 1\right)}\left[\left|\psi^{\prime}\right|\left|\frac{\partial \bar{v}}{\partial x}\right|^{m-2}\right]^{\frac{m}{m-1}} d x .
\end{gathered}
$$

Applying Hölder's inequality to the second integral in the right-hand side of this inequality and using inequality (27), we get

$$
I_{2} \leqslant \varepsilon \int_{B\left(x_{i}^{(s)}, 1\right)}\left|\frac{\partial(\bar{v}-\hat{v})}{\partial x}\right|^{m} d x+C|\bar{k}-\hat{k}|^{\frac{m}{m-1}}|\bar{k}|^{\frac{m(m-2)}{m-1}}\left[d_{i}^{(s)}\right]^{n-m} .
$$

We estimate $I_{3}$ analogously. Thus from (32)-(34), with $\varepsilon$ sufficiently small, we obtain (30).

Next we prove (31). We substitute the test functions

$$
\varphi(x)=\frac{1}{\bar{k}_{\alpha}} v_{i \alpha}^{(s)}(x, \bar{k})-\psi\left(\frac{x-x_{i}^{(s)}}{d_{i}^{(s)}}\right) \vec{e}^{(\alpha)}
$$


and

$$
\varphi(x)=\frac{1}{\hat{k}_{\alpha}} v_{i \alpha}^{(s)}(x, \hat{k})-\psi\left(\frac{x-x_{i}^{(s)}}{d_{i}^{(s)}}\right) \vec{e}^{(\alpha)},
$$

in the integral identity (20) corresponding to $v_{i}^{(s)}(x, \bar{k})$ and $v_{i}^{(s)}(x, \hat{k})$ respectively, the function $v_{i \alpha}^{(s)}(x, \vec{k})$ are defined as in the formulation of condition C. Subtracting the resulting equations from each other, we get

$$
\begin{aligned}
& \sum_{j=1}^{N} \int_{B\left(x_{i}^{(s)}, 1\right)}\left\{A^{j}\left(x, \frac{\partial \bar{v}}{\partial x}\right) \frac{1}{\bar{k}_{\alpha}} \frac{\partial v_{i \alpha}^{(s)}(x, \bar{k})}{\partial x}-A^{j}\left(x, \frac{\partial \hat{v}}{\partial x}\right) \frac{1}{\hat{k}_{\alpha}} \frac{\partial v_{i \alpha}^{(s)}(x, \hat{k})}{\partial x}\right\} d x \\
& \quad \leqslant \sum_{j=1}^{N} \int_{B\left(x_{i}^{(s)}, 1\right)}\left|A^{j}\left(x, \frac{\partial \bar{v}}{\partial x}\right)-A^{j}\left(x, \frac{\partial \hat{v}}{\partial x}\right)\right|\left|\frac{\partial \psi}{\partial x}\right| d x=J_{1} .
\end{aligned}
$$

Let us estimate $J_{1}$. From condition 2) and Hölder's inequality, we have

$$
\begin{aligned}
J_{1} \leqslant & C\left\{\int_{B\left(x_{i}^{(s)}, 1\right)}\left|\frac{\partial(\bar{v}-\hat{v})}{\partial x}\right|^{m} d x\right\}^{\frac{1}{m}}\left[d_{i}^{(s)}\right]^{\frac{n(m-1)}{m}-1} \\
& +C\left[\left\{\int_{B\left(x_{i}^{(s)}, 1\right)}\left|\frac{\partial \bar{v}}{\partial x}\right|^{m} d x\right\}^{\frac{m-2}{m}}+\left\{\int_{B\left(x_{i}^{(s)}, 1\right)}\left|\frac{\partial \hat{v}}{\partial x}\right|^{m} d x\right\}^{\frac{m-2}{m}}\right] \\
& \times\left\{\int_{B\left(x_{i}^{(s)}, 1\right)}\left|\frac{\partial(\bar{v}-\hat{v})}{\partial x}\right|^{m} d x\right\}^{\frac{1}{m}}\left[d_{i}^{(s)}\right]^{\frac{n-m}{m}} .
\end{aligned}
$$

Thus from (27) and (30), we get

$$
J_{1} \leqslant C|\bar{k}-\hat{k}|^{\frac{1}{m-1}}\left[d_{i}^{(s)}\right]^{n-m} .
$$

(31) immediately follows from this inequality and inequality (35). The theorem is proved.

\section{Asymptotic expansion of the sequence of solutions}

In this section we construct an asymptotic expansion for the sequence of solutions $u_{s}$ of the problem (1)-(2) with a remainder. We prove the convergence of the remainder term to zero in appropriate topologies. Let us introduce the sequence

$$
\rho_{i}^{(s)}=\max \left\{\left(1+\frac{1}{2 C_{1}}\right) d_{i}^{(s)}, \frac{1}{2 C_{3}}\left[r_{i}^{(s)}\right]^{\frac{n}{n-m}} \ln ^{2} r_{i}^{(s)}\right\},
$$

where $C_{1}$ is the constant from hypothesis $\mathrm{B} 1$ and

$$
C_{3}=\max _{0 \leqslant t \leqslant \operatorname{diam} \Omega}\left\{t^{\frac{m}{n-m}} \ln ^{2} t\right\} .
$$


A simple calculation shows that

$$
\rho_{i}^{(s)} \leqslant d_{i}^{(s)}+\frac{r_{i}^{(s)}}{2}
$$

and from the definition of the numbers $d_{i}^{(s)}$ and $r_{i}^{(s)}$, it follows that the balls $B\left(x_{i}^{(s)}, d_{i}^{(s)}+\right.$ $\left.r_{i}^{(s)} / 2\right)(i=1, \ldots, I(s))$ are pairwise disjoint. Let $\theta_{1}, \theta_{2}$ and $\theta_{3}$ be some numbers such that $0<\theta_{3}<\theta_{2}<\theta_{1}<1$. We consider the infinitely differentiable functions $\chi_{j}$, $j=1,2$, satisfying the conditions: $0 \leqslant \chi_{j}(t) \leqslant 1, \chi_{j}(t)=1$ if $t<\theta_{j+1}, \chi_{j}(t)=0$ if $t>\theta_{j}, \chi_{j}^{\prime}(t)<\infty(j=1,2)$. We introduce the functions

$$
\psi_{i}^{(s)}(x)=\chi_{1}\left(\frac{\left|x-x_{i}^{(s)}\right|}{\rho_{i}^{(s)}}\right), \varphi_{i}^{(s)}(x)=\chi_{2}\left(\frac{\left|x-x_{i}^{(s)}\right|}{\rho_{i}^{(s)}}\right) ; \quad i=1, \ldots, I(s) .
$$

We set

$$
\begin{aligned}
& I_{s}^{\prime}=\left\{i=1, \ldots, I(s): \theta_{1} d_{i}^{(s)} \geqslant \frac{1}{2 C_{3}}\left[r_{i}^{(s)}\right]^{\frac{n}{n-m}} \ln ^{2} r_{i}^{(s)}\right\}, \\
& I_{s}^{\prime \prime}=\left\{i=1, \ldots, I(s): \theta_{1} d_{i}^{(s)}<\frac{1}{2 C_{3}}\left[r_{i}^{(s)}\right]^{\frac{n}{n-m}} \ln ^{2} r_{i}^{(s)}\right\}
\end{aligned}
$$

We have

LEMMA 7. - If conditions $\mathrm{B} 1$ and $\mathrm{B} 2$ are satisfied, then

$$
\begin{aligned}
& \lim _{s \rightarrow \infty} \sum_{i \in I_{s}^{\prime}}\left[d_{i}^{(s)}\right]^{n-m}=0, \\
& \lim _{s \rightarrow \infty} \sum_{i \in I_{s}^{\prime \prime}}\left[\rho_{i}^{(s)}\right]^{n}=0 .
\end{aligned}
$$

Proof. - We have

$$
\sum_{i \in I_{s}^{\prime}}\left[d_{i}^{(s)}\right]^{n-m} \leqslant C \max _{1 \leqslant i \leqslant I(s)}\left\{\frac{1}{\left[\ln r_{i}^{(s)}\right]^{2 \frac{n-m}{m-1}}}\right\} \sum_{i \in I_{s}^{\prime}}\left[\frac{\left[d_{i}^{(s)}\right]^{m(n-m)}}{\left[r_{i}^{(s)}\right]^{n}}\right]^{\frac{1}{m-1}} .
$$

By condition B2, (36) follows from a passage to the limit as $s \rightarrow \infty$ in the above inequality; here we have made use of the definition of the set $I_{s}^{\prime}$.

For the proof of the relation (37), we note that since the balls $B\left(x_{i}^{(s)}, d_{i}^{(s)}+r_{i}^{(s)} / 2\right)$, $i=1, \ldots, I(s)$ are pairwise disjoint and $\Omega$ is bounded, it follows that

$$
\sum_{i=1}^{I(s)}\left[r_{i}^{(s)}\right]^{n} \leqslant C
$$

$C$ is a constant independent of $s$. We have, by the definition of the set $I_{s}^{\prime \prime}$,

$$
\sum_{i \in I_{s}^{\prime \prime}}\left[\rho_{i}^{(s)}\right]^{n} \leqslant\left[\frac{1}{2 C_{3}}\right]^{n} \max _{1 \leqslant i \leqslant I(s)}\left\{\left[r_{i}^{(s)}\right]^{\frac{n m}{n-m}} \ln ^{2 n} r_{i}^{(s)}\right\} \sum_{i \in I_{s}^{\prime \prime}}\left[r_{i}^{(s)}\right]^{n}
$$


A passage to the limit in both sides of this inequality and the relation (38) yield (37). The lemma is proved.

As an immediate consequence of Lemma 7, we have

$$
\lim _{s \rightarrow \infty} \sum_{i=1}^{I(s)}\left[\rho_{i}^{(s)}\right]^{n}=0 .
$$

Further we see that

$$
\sum_{i=1}^{I(s)}\left[d_{i}^{(s)}\right]^{n-m}<C
$$

$C$ is independent of $s$. Indeed by Hölder's inequality, we have

$$
\sum_{i=1}^{I(s)}\left[d_{i}^{(s)}\right]^{n-m} \leqslant\left\{\sum_{i=1}^{I(s)}\left[\frac{\left[d_{i}^{(s)}\right]^{n-m}}{\left[r_{i}^{(s)}\right]^{\frac{n}{m}}}\right]^{\frac{m}{m-1}}\right\}^{\frac{m-1}{m}}\left\{\sum_{i=1}^{I(s)}\left[r_{i}^{(s)}\right]^{n}\right\}^{\frac{1}{m}} .
$$

Thus, (40) is an immediate consequence of condition B2 and relation (39). Inequality (40) implies in particular that

$$
\lim _{s \rightarrow \infty} \sum_{i=1}^{I(s)}\left[d_{i}^{(s)}\right]^{n}=0
$$

i.e., the set $\bigcup_{i=1}^{I(s)} F_{i}^{(s)}$ vanishes as $s \rightarrow \infty$.

Now we are in a position to construct the asymptotic expansion of the sequence of solutions $u_{s}$ of the problem (1)-(2). We assume that $u_{s}$ satisfies the inequalities (7) and (8) and converges weakly to the vector-function $u_{0} \in f+\stackrel{\circ}{W_{m}^{1}}(\Omega, N)$ with $f \in$ $W_{q}^{1}(\Omega, N)$ and $q>n$. We denote the means of the functions $u_{0}$ and $f$ over the balls $B_{i}^{(s)}=: B\left(x_{i}^{(s)}, 1\right)$ by

$$
\begin{aligned}
& u_{i}^{(s)}=\oint_{B_{i}^{s}} u_{0}(x) d x=: \frac{1}{\operatorname{meas} B_{i}^{(s)}} \int_{B_{i}^{(s)}} u_{0}(x) d x, \\
& f_{i}^{(s)}=\frac{1}{\operatorname{meas} B_{i}^{(s)}} \int_{B_{i}^{(s)}} f(x) d x,
\end{aligned}
$$

respectively. In the sequel we set $D_{i}^{(s)}=: B\left(x_{i}^{(s)}, \theta_{2} \rho_{i}^{(s)}\right)$.

We seek a solution of problem (1)-(2) in the form

$$
u_{s}(x)=u_{0}(x)+\sum_{k=1}^{4} R_{s}^{(k)}(x)+w_{s}(x),
$$

where 


$$
\begin{gathered}
R_{s}^{(1)}(x)=\sum_{i \in I_{s}^{\prime}}\left\{\left[u_{i}^{(s)}-u_{0}(x)\right]+\left[f(x)-f_{i}^{(s)}\right]\right\} \psi_{i}^{(s)}(x) \\
R_{s}^{(2)}=\sum_{i \in I_{s}^{\prime \prime}}\left\{\left[u_{i}^{(s)}-u_{0}(x)\right]+\left[f(x)-f_{i}^{(s)}\right]\right\} \varphi_{i}^{(s)}(x) \\
R_{s}^{(3)}=\sum_{i \in I_{s}^{\prime}} v_{i}^{(s)}\left(x, f_{i}^{(s)}-u_{i}^{(s)}\right) \varphi_{i}^{(s)}(x) \\
R_{s}^{(4)}=\sum_{i \in I_{s}^{\prime \prime}} v_{i}^{(s)}\left(x, f_{i}^{(s)}-u_{i}^{(s)}\right) \varphi_{i}^{(s)}(x)
\end{gathered}
$$

where $v_{i}^{(s)}\left(x, f_{i}^{(s)}-u_{i}^{(s)}\right)$ is a solution of problem (21)-(22) with $k=\left|f_{i}^{(s)}-u_{i}^{(s)}\right|$ and $\vec{e}=\frac{f_{i}^{(s)}-u_{i}^{(s)}}{\left|f_{i}^{(s)}-u_{i}^{(s)}\right|}, w_{s}$ is the remainder term whose behavior will be dealt with in the following corrector result which gives a justification of the expansion (41). For simplicity we shall write $v_{i}^{(s)}\left(x, f_{i}^{(s)}-u_{i}\right)$ as $v_{i}^{(s)}$ and indicate "converge to" by $\rightarrow$.

THEOREM 8. - Let the conditions of Theorem 3 be satisfied. Then

1. $R_{s}^{(k)} \rightarrow 0$ strongly in $W_{m}^{1}(\Omega, N)(k=1,2,3)$.

2. $R_{s}^{(4)} \rightarrow 0$ weakly in $W_{m}^{1}(\Omega, N)$ and strongly in $W_{p}^{1}(\Omega, N)$ for all $p \in(1, m)$.

3. $w_{s} \rightarrow 0$ strongly in $W_{m}^{1}(\Omega, N)$.

Proof. -

Proof of 1 . We have

$$
\left\|R_{s}^{(1)}\right\|_{L_{m}(\Omega, N)} \leqslant \sum_{i \in I_{s}^{\prime}} \int_{\Omega}\left[\left|u_{i}^{(s)}-u_{0}(x)\right|^{m}+\left|f_{i}^{(s)}-f(x)\right|^{m}\right]\left|\psi_{i}^{(s)}\right|^{m} d x .
$$

Theorem 2 implies that $u_{0}$ is uniformly bounded in $\Omega$. Thus using inequality (9), Poincare inequality and the definition of $I_{s}^{\prime}$, we get

$$
\left\|R_{s}^{(1)}\right\|_{L_{m}(\Omega, N)} \leqslant C \sum_{i \in I_{s}^{\prime}}\left[d_{i}^{(s)}\right]^{n-m}
$$

Next

$$
\begin{aligned}
\left\|\frac{\partial R_{s}^{(1)}}{\partial x}\right\|_{L_{m}(\Omega, N)} \leqslant & C \sum_{i \in I_{s}^{\prime} \Omega} \int_{\Omega}\left\{\left|u_{i}^{(s)}-u_{0}(x)\right|^{m}+\left|f_{i}^{(s)}-f(x)\right|^{m}\right]\left|\frac{\partial \psi_{i}^{(s)}}{\partial x}\right|^{m} \\
& \left.+\left[\left|\frac{\partial u_{0}}{\partial x}\right|^{m}+\left|\frac{\partial f}{\partial x}\right|^{m}\right]\left|\psi_{i}^{(s)}(x)\right|^{m}\right\} d x \\
\leqslant & C\left\{\sum_{i \in I_{s}^{\prime}}\left[d_{i}^{(s)}\right]^{n-m}+\int_{\Omega}\left[\left|\frac{\partial u_{0}}{\partial x}\right|^{m}+\left|\frac{\partial f}{\partial x}\right|^{m}\right] \sum_{i \in I_{s}^{\prime}}\left|\psi_{i}^{(s)}(x)\right|^{m} d x\right\},
\end{aligned}
$$


where as above, we have used Theorem 2, inequality (9) and the definition of $I_{s}^{\prime}$. The function $\Phi_{s}(x)=\sum_{i \in I_{s}^{\prime}}\left|\psi_{i}^{(s)}(x)\right|^{m}$ is bounded and by Poincare's inequality, we have

$$
\int_{\Omega} \Phi_{s}(x) d x \leqslant C \sum_{i \in I_{s}^{\prime}} \int_{D_{i}^{(s)}}\left|\frac{\partial \psi_{i}^{(s)}}{\partial x}\right|^{m} d x \leqslant C \sum_{i \in I_{s}^{\prime}}\left[d_{i}^{(s)}\right]^{n-m} .
$$

Thus, we get

$$
\lim _{s \rightarrow \infty}\left\|\frac{\partial R_{s}^{(1)}}{\partial x}\right\|_{L_{m}(\Omega, N)} \leqslant C \sum_{i \in I_{s}^{\prime}}\left[d_{i}^{(s)}\right]^{n-m} .
$$

Passing to the limit in (42) and (43) and using (36), we obtain that $R_{1}^{(s)}$ converges to zero strongly in $W_{m}^{1}(\Omega, N)$.

In a similar fashion we show that

$$
\lim _{s \rightarrow \infty}\left\|R_{s}^{(2)}\right\|_{W_{m}^{1}(\Omega, N)}=0
$$

Next we have

$$
\begin{aligned}
\left\|R_{s}^{(3)}\right\|_{L_{m}(\Omega, N)} & \leqslant \sum_{i \in I_{s}^{\prime}} \int_{\Omega}\left|v_{i}^{(s)}\left(x, f_{i}^{(s)}-u_{i}^{(s)}\right)\right|^{m}\left|\varphi_{i}^{(s)}\right|^{m} d x \\
& \leqslant C \sum_{i \in I_{s}^{\prime}} \int_{\Omega}\left|\frac{\partial v_{i}^{(s)}}{\partial x}\right|^{m} d x \leqslant C \sum_{i \in I_{s}^{\prime}}\left[d_{i}^{(s)}\right]^{n-m},
\end{aligned}
$$

where we have used Poincare inequality and inequality (27) from Theorem 5.

Next we have

$$
\begin{aligned}
\left\|\frac{\partial R_{s}^{(3)}}{\partial x}\right\|_{L_{m}(\Omega, N)} & \leqslant C \sum_{i \in I_{s}^{\prime}} \int_{\Omega}\left[\left|v_{i}^{(s)}(x)\right|^{m}\left|\frac{\partial \varphi_{i}^{(s)}}{\partial x}\right|^{m}+\left|\frac{\partial v_{i}^{(s)}}{\partial x}\right|\left|\varphi_{i}^{(s)}(x)\right|^{m}\right] d x \\
& \leqslant C \sum_{i \in I_{s}^{\prime}}\left[d_{i}^{(s)}\right]^{n-m} .
\end{aligned}
$$

Here we have used the boundedness of $v_{i}^{(s)}$, the definition of $\varphi_{i}^{(s)}$ and the inequality (27) from Theorem 5. Combining this inequality and the previous one and taking account of (36), we get

$$
\lim _{s \rightarrow \infty}\left\|R_{s}^{(3)}\right\|_{W_{m}^{1}(\Omega, N)}=0 .
$$

Part 1 of the theorem is proved.

Proof of 2. By Theorem 5 (inequality (27)) and the boundedness of $v_{i}^{(s)}$ we have

$$
\left\|R_{s}^{(4)}\right\|_{W_{m}^{1}(\Omega, N)}^{m} \leqslant C \sum_{i \in I_{s}^{\prime \prime}}\left\{\left[d_{i}^{(s)}\right]^{n-m}+\left[\rho_{i}^{(s)}\right]^{n}\right\} .
$$

Thus the relation (40) and (39) imply

$$
\lim _{s \rightarrow \infty}\left\|R_{s}^{(4)}\right\|_{W_{m}^{1}(\Omega, N)}<\infty .
$$


We deduce from here that $R_{s}^{(4)}$ converges weakly in $W_{m}^{1}(\Omega, N)$. Next by Hölder's inequality and (46), we have for $1<p<m$

$$
\left\|R_{s}^{(4)}\right\|_{W_{p}^{1}(\Omega, N)} \leqslant C\left\|R_{s}^{(4)}\right\|_{W_{m}^{1}(\Omega, N)}\left\{\sum_{i \in I_{s}^{\prime \prime}} \operatorname{meas} D_{i}^{(s)}\right\}^{\frac{1}{p}-\frac{1}{m}} \leqslant C\left\{\sum_{i \in I_{s}^{\prime \prime}}\left[\rho_{i}^{(s)}\right]^{n}\right\}^{\frac{1}{p}-\frac{1}{m}} ;
$$

we have used the fact that $\varphi_{i}^{(s)}(x)=0$ outside $\bigcup_{i=1}^{I(s)} D_{i}^{(s)}$. Hence by relation (37), we get

$$
\lim _{s \rightarrow \infty}\left\|R_{s}^{(4)}\right\|_{W_{p}^{1}(\Omega, N)}=0 .
$$

This implies the claimed strong convergence of $R_{s}^{(4)}$ to zero in $W_{p}^{1}(\Omega, N)$.

Proof of 3 . From the assertions 1 and 2 of the theorem and the weak convergence of $u_{s}$ to $u$ in $W_{m}^{1}(\Omega, N)$, it clearly follows that $w_{s}$ weakly converges to zero in $W_{m}^{1}(\Omega, N)$. Furthermore, since the sequence $w_{s}$ is uniformly bounded, we also have that $w_{s}$ strongly converges to zero in any $L_{r}(\Omega, N)$ for all $r<\infty$ by Sobolev embedding's theorem.

We have $w_{s} \in \stackrel{\circ}{W}_{m}^{1}\left(\Omega^{(s)}, N\right)$; in particular $w_{s}(x)=0$ in $\bigcup_{i}^{I(s)} F_{i}^{(s)}$. Hence we can substitute $\varphi(x)=w_{s}(x)=\left(w_{s 1}(x), \ldots, w_{s N}(x)\right)$ in the integral identity (6) and get

$$
\sum_{j=1}^{N} \int_{\Omega} A^{j}\left(x, \frac{\partial u_{s}}{\partial x}\right) \frac{\partial w_{s j}(x)}{\partial x} d x=0 .
$$

We rewrite the left-hand side of (48) as

$$
\sum_{j=1}^{N} \int_{\Omega^{(s)}} A^{j}\left(x, \frac{\partial u_{s}}{\partial x}\right) \frac{\partial w_{s j}(x)}{\partial x} d x=I_{1}^{(s)}+I_{2}^{(s)}+I_{3}^{(s)}+I_{4}^{(s)},
$$

where

$$
\begin{gathered}
I_{1}^{(s)}=\int_{\Omega} \sum_{j=1}^{N}\left[A^{j}\left(x, \frac{\partial u_{s}}{\partial x}\right)-A^{j}\left(x, \frac{\partial u_{s}}{\partial x}-\frac{\partial w_{s}}{\partial x}\right)\right] \frac{\partial w_{s j}(x)}{\partial x} d x \\
I_{2}^{(s)}=\int_{\Omega} \sum_{j=1}^{N}\left[A^{j}\left(x, \frac{\partial u_{s}}{\partial x}-\frac{\partial w_{s}}{\partial x}\right)-A^{j}\left(x, \frac{\partial u_{0}}{\partial x}+\frac{\partial R_{s}^{(4)}}{\partial x}\right)\right] \frac{\partial w_{s j}(x)}{\partial x} d x, \\
I_{3}^{(s)}=\int_{\Omega} \sum_{j=1}^{N}\left[A^{j}\left(x, \frac{\partial u_{0}}{\partial x}+\frac{\partial R_{s}^{(4)}}{\partial x}\right)-A^{j}\left(x, \frac{\partial R_{s}^{(4)}}{\partial x}\right)\right] \frac{\partial w_{s j}(x)}{\partial x} d x, \\
I_{4}^{(s)}=\int_{\Omega} \sum_{j=1}^{N} A^{j}\left(x, \frac{\partial R_{s}^{(4)}}{\partial x}\right) \frac{\partial w_{s j}(x)}{\partial x} d x .
\end{gathered}
$$

By condition (4) on the $A^{j}$,s, we obtain the estimate

$$
I_{1}^{(s)} \geqslant v_{2} \int_{\Omega}\left|\frac{\partial w_{s}}{\partial x}\right|^{m} d x
$$


Inequality (5) and Hölder's inequality yield

$$
\begin{aligned}
I_{2}^{(s)} \leqslant & C\left\{\int_{\Omega}\left[1+\left|\frac{\partial u_{s}}{\partial x}\right|+\left|\frac{\partial u_{0}}{\partial x}\right|+\left|\frac{\partial R_{s}^{(4)}}{\partial x}\right|+\left|\frac{\partial w_{s}}{\partial x}\right|\right]^{m} d x\right\}^{\frac{m-2}{m}} \\
& \times\left\{\int_{\Omega}\left[\sum_{k=1}^{3}\left|\frac{\partial R_{s}^{(k)}}{\partial x}\right|\right]^{m} d x\right\}^{\frac{1}{m}}\left\{\int_{\Omega}\left|\frac{\partial w_{s}}{\partial x}\right|^{m}\right\}^{\frac{1}{m}} .
\end{aligned}
$$

From relations (43)-(45) we deduce from this inequality that

$$
\lim _{s \rightarrow \infty} I_{2}^{(s)}=0 .
$$

For the estimation of $I_{3}^{(s)}$, we note that since $R_{s}^{(4)}(x)=0$ in $K^{(s)}=\Omega \backslash \bigcup_{i=1}^{I^{\prime \prime}(s)} D_{i}^{(s)}$, we have the following representation

$$
\begin{aligned}
I_{3}^{(s)}= & \int_{K^{(s)}} \sum_{j=1}^{N} A^{j}\left(x, \frac{\partial u_{0}}{\partial x}\right) \frac{\partial w_{s j}}{\partial x} d x \\
& +\sum_{j=1}^{N} \sum_{i \in I_{s}^{\prime \prime} D_{i}^{(s)}}\left[A^{j}\left(x, \frac{\partial u_{0}}{\partial x}+\frac{\partial R_{s}^{(4)}}{\partial x}\right)-A^{j}\left(x, \frac{\partial R_{s}^{(4)}}{\partial x}\right)\right] \frac{\partial w_{s j}}{\partial x} d x \\
\equiv & I_{31}^{(s)}+I_{32}^{(s)} .
\end{aligned}
$$

By inequality (5) and Young's inequality, we have

$$
\int_{K^{(s)}}\left|A^{j}\left(x, \frac{\partial u_{0}}{\partial x}\right)\right|^{\frac{m}{m-1}} d x \leqslant C \int_{\Omega}\left|\frac{\partial u_{0}}{\partial x}\right|^{m} d x .
$$

This implies that $\left|A^{j}\left(x, \frac{\partial u_{0}}{\partial x}\right)\right| \in L_{\frac{m}{m-1}}(\Omega)$. Hence from the weak convergence of $w_{s}$ to zero in $W_{m}^{1}(\Omega, N)$, we obtain that $I_{31}^{(s)}$ converges to zero as $s \rightarrow \infty$. Further by inequality (5) and Hölder's inequality, we have

$$
I_{32}^{(s)} \leqslant C\left\{\int_{\Omega}\left[1+\left|\frac{\partial u_{0}}{\partial x}\right|+\left|\frac{\partial R_{s}^{(4)}}{\partial x}\right|\right]^{m} d x\right\}^{\frac{m-2}{m}}\left\{\int\left|\frac{\partial w_{s}}{\partial x}\right|^{m} d x\right\}^{\frac{1}{m}}\left\{\sum_{i \in I_{s}^{\prime \prime} D_{i}^{(s)}}\left|\frac{\partial u_{0}}{\partial x}\right|^{m} d x\right\}^{\frac{1}{m}} .
$$

In view of (39) and the absolute continuity of integrals, the last integral converges to zero as $s \rightarrow \infty$; the two previous are bounded. Thus $I_{32}^{(s)}$ converges to zero. Hence we obtain

$$
\lim _{s \rightarrow \infty} I_{3}^{(s)}=0 .
$$

Now we proceed to the estimation of $I_{4}^{(s)}$. It is technically the most complicated part of the proof of the theorem. But firstly, let us introduce a new test function. Let $\lambda \in\left(0, \theta_{3}\right)$ and let us consider the functions $\chi_{i}^{(s)} \in \mathbf{C}_{o}^{\infty}(\Omega)$ such that $0 \leqslant \chi_{i}^{(s)}(x) \leqslant 1$, 
$\chi_{i}^{(s)}(x)=1$ in $B\left(x_{i}^{(s)}, \lambda \rho_{i}^{(s)}\right), \chi_{i}^{(s)}(x)=0$ outside $B\left(x_{i}^{(s)}, \theta_{3} \rho_{i}^{(s)}\right)$ and $\left|\frac{\partial \chi_{i}^{(s)}}{\partial x}\right| \leqslant \frac{C}{\rho_{i}^{(s)}}$. Using these functions, we rewrite $I_{4}^{(s)}$ as follows

$$
I_{4}^{(s)}=I_{41}^{(s)}+I_{42}^{(s)},
$$

where

$$
I_{41}^{(s)}=\sum_{i \in I_{s^{\prime \prime}} D_{i}^{(s)}} \sum_{j=1}^{N} A^{j}\left(x, \frac{\partial v_{i}^{(s)}}{\partial x}\right) \frac{\partial\left[w_{s j} \chi_{i}^{(s)}\right]}{\partial x} d x
$$

and

$$
I_{42}^{(s)}=\sum_{i \in I_{s}^{\prime \prime}} \int_{D_{i}^{(s)}} \sum_{j=1}^{N} A^{j}\left(x, \frac{\partial\left[\varphi_{i}^{(s)} v_{i}^{(s)}\right]}{\partial x}\right) \frac{\partial\left[w_{s j}\left(1-\chi_{i}^{(s)}\right)\right]}{\partial x} d x .
$$

Let $s$ be sufficiently large so that $\theta_{3} \rho_{i}^{(s)}<1$. Then $I_{41}^{(s)}=0$ by the definition of the functions $v_{i}^{(s)}\left(x, f_{i}^{(s)}-u_{i}^{(s)}\right)$. For simplicity let us denote the ball $B\left(x_{i}^{(s)}, \lambda \rho_{i}^{(s)}\right)$ by $G_{i}^{(s)}$. Appealing to Hölder's and Young's inequalities and using inequality (5) we get

$$
\begin{aligned}
I_{42}^{(s)} \leqslant & C \sum_{i \in I_{s}^{\prime \prime}}\left\{\int_{D_{i}^{(s)}}\left|\frac{\partial\left[w_{s j}\left(1-\chi_{i}^{(s)}\right)\right]}{\partial x}\right|^{m} d x\right\}^{\frac{1}{m}} \\
& \times\left\{\left[\rho_{i}^{(s)}\right]^{n}+\int_{D_{i}^{(s)} \backslash G_{i}^{(s)}}\left|\frac{\partial\left[\varphi_{i}^{(s)} v_{i}^{(s)}\right]}{\partial x}\right|^{m} d x\right\} .
\end{aligned}
$$

Let $m_{i}^{(s)}=\max _{x \in D_{i}^{(s)} \backslash G_{i}^{(s)}}\left|v_{i}^{(s)}(x)\right|$. By Theorem 5 (inequality (29)), we have

$$
m_{i}^{(s)} \leqslant C\left[\frac{d_{i}^{(s)}}{\rho_{i}^{(s)}}\right]^{\frac{n-m}{m-1}}
$$

Thus using the properties of the functions $\varphi_{i}^{(s)}$ and the inequality (28) in Theorem 5, we get

$$
\begin{aligned}
\int_{D_{i}^{(s)} \backslash G_{i}^{(s)}}\left|\frac{\partial\left[\varphi_{i}^{(s)} v_{i}^{(s)}\right]}{\partial x}\right|^{m} d x & \leqslant C\left\{\int_{E_{m_{i}^{(s)}}}\left|\frac{\partial v_{i}^{(s)}}{\partial x}\right|^{m} d x+\int_{D_{i}^{(s)} \backslash G_{i}^{(s)}}\left|v_{i}^{(s)}(x)\right|^{m}\left|\frac{\partial \varphi_{i}^{(s)}}{\partial x}\right|^{m} d x\right\} \\
& \leqslant C\left\{m_{i}^{(s)}\left[d_{i}^{(s)}\right]^{n-m}+\left[m_{i}^{(s)}\right]^{m}\left[\rho_{i}^{(s)}\right]^{n-m}\right\} \\
& \leqslant C\left[\frac{\left[d_{i}^{(s)}\right]^{m}}{\rho_{i}^{(s)}}\right]^{\frac{n-m}{m-1}} .
\end{aligned}
$$

For the estimation of the first factor in the last inequality in (54), we need the following Poincare's inequality from [17, Lemma 1.4, Chap. 8]. Let $1<p<n$, then for any 
function $u \in W_{p}^{1}(B(0, r))$ and any numbers $\rho_{1}$ and $\rho_{2}$ such that $0 \leqslant \rho_{1}<\rho_{2} \leqslant \frac{r}{2}$, the inequality

$$
\int_{K\left(\rho_{1}, \rho_{2}\right)}|u(x)|^{p} d x \leqslant C\left\{\left(\rho_{2}^{p}-\rho_{1}^{p}\right) \int_{K\left(\rho_{1}, r\right)}\left|\frac{\partial u}{\partial x}\right|^{p} d x+\frac{\rho_{2}^{n}-\rho_{1}^{n}}{r^{n}} \int_{K\left(\frac{r}{2}, r\right)}|u(x)|^{p} d x\right\},
$$

holds with the constant $C$ depending only on $n$ and $p ; K(a, b)=\{x: a<|x|<b\}$.

We have

$$
\int_{D_{i}^{(s)}}\left|\frac{\partial\left[w_{s j}\left(1-\chi_{i}^{(s)}\right)\right]}{\partial x}\right|^{m} d x \leqslant C\left\{\int_{D_{i}^{(s)}}\left|\frac{\partial w_{s j}}{\partial x}\right|^{m} d x+\left[\rho_{i}^{(s)}\right]^{-m} \int_{D_{i}^{(s)}}\left|w_{s j}\right|^{m} d x\right\} .
$$

Let $\bar{r}_{i}^{(s)}=d_{i}^{(s)}+\frac{r_{i}^{(s)}}{2}$. Thus $D_{i}^{(s)} \subset B\left(x_{i}^{(s)}, \bar{r}_{i}^{(s)}\right) \subset B\left(x_{i}^{(s)}, 2 \bar{r}_{i}^{(s)}\right)$. By inequality (56) it follows that

$$
\begin{aligned}
\int_{D_{i}^{(s)}}\left|w_{s j}(x)\right|^{m} d x \leqslant & C\left\{\left[\theta_{2} \rho_{i}^{(s)}\right]^{m} \int_{B\left(x_{i}^{(s)}, \bar{r}_{i}^{(s)}\right)}\left|\frac{\partial w_{s j}}{\partial x}\right|^{m} d x \frac{\left[\theta_{2} \rho_{i}^{(s)}\right]^{n}}{\left[r_{i}^{(s)}+2 d_{i}^{(s)}\right]^{n}}\right. \\
& \left.\times \int_{B\left(x_{i}^{(s)}, \bar{r}_{i}^{(s)}\right)}\left|w_{s j}(x)\right|^{m} d x\right\} .
\end{aligned}
$$

Thus we obtain

$$
\begin{aligned}
& \int_{D_{i}^{(s)}}\left|\frac{\partial\left[w_{s j}\left(1-\chi_{i}^{(s)}\right)\right]}{\partial x}\right|^{m} d x \\
& \quad \leqslant C\left\{\int_{B\left(x_{i}^{(s)}, \bar{r}_{i}^{(s)}\right)}\left|\frac{\partial w_{s j}}{\partial x}\right|^{m} d x+\frac{\left[\rho_{i}^{(s)}\right]^{n-m}}{\left[r_{i}^{(s)}\right]^{n}} \int_{B\left(x_{i}^{(s)}, \bar{r}_{i}^{(s)}\right)}\left|w_{s j}(x)\right|^{m} d x\right\} .
\end{aligned}
$$

From this inequality and (54)-(55), we get

$$
\begin{aligned}
I_{42}^{(s)} \leqslant & C_{1}\left\{\sum_{i \in I_{s}^{\prime \prime}}\left[\frac{\left[d_{i}^{(s)}\right]^{m}}{\rho_{i}^{(s)}}\right]^{\frac{n-m}{m-1}}+\sum_{i \in I_{s}^{\prime \prime}}\left[\rho_{i}^{(s)}\right]^{n}\right\}^{\frac{m-1}{m}}\left\{\int\left|\frac{\partial w_{s}}{\partial x}\right|^{m} d x\right\}^{\frac{1}{m}} \\
& +C_{2}\left\{\sum_{i \in I_{s}^{\prime \prime}}\left[\frac{\left[d_{i}^{(s)}\right]^{m(n-m)}}{\left[r_{i}^{(s)}\right]^{n}}\right]^{\frac{1}{m-1}}+\sum_{i \in I_{s}^{\prime \prime}}\left[\rho_{i}^{(s)}\right]^{n}\left[\frac{\left[\rho_{i}^{(s)}\right]^{n-m}}{\left[r_{i}^{(s)}\right]^{n}}\right]^{\frac{1}{m-1}}\right\}^{\frac{m-1}{m}} \\
& \times\left\{\int_{\Omega}\left|w_{s j}(x)\right|^{m} d x\right\} .
\end{aligned}
$$

By the definition of the set $I_{s}^{\prime \prime}$, we get

$$
A_{s}=\sum_{i \in I_{s}^{\prime \prime}}\left[\frac{\left[d_{i}^{(s)}\right]^{m}}{\rho_{i}^{S}}\right]^{\frac{n-m}{m-1}} \leqslant C \max _{1 \leqslant i \leqslant I(s)} \frac{1}{\ln ^{2 \frac{n-m}{m-1}} r_{i}^{(s)}} \sum_{i \in I_{s}^{\prime \prime}}\left[\frac{\left[d_{i}^{(s)}\right]^{m(n-m)}}{\left[r_{i}^{(s)}\right]^{n}}\right]^{\frac{1}{m-1}} .
$$


Passing to the limit in this inequality we get from the conditions $\mathrm{B} 1$ and $\mathrm{B} 2$ that $A_{s}$ converges to zero as $s \rightarrow \infty$. Thus in view of (37), we obtain that the first term in the right-hand side of (57) converges to zero as $s \rightarrow \infty$.

Further, we have

$$
E_{s}=\sum_{i \in I_{s}^{\prime \prime}}\left[\rho_{i}^{(s)}\right]^{n}\left[\frac{\left[\rho_{i}^{(s)}\right]^{n-m}}{\left[r_{i}^{(s)}\right]^{n}}\right]^{\frac{1}{m-1}} \leqslant \max _{1 \leqslant i \leqslant I(s)}\left\{\left[r_{i}^{(s)}\right]^{\frac{n m}{n-m}}\left[\ln r_{i}^{(s)}\right]^{2\left[n+\frac{n-m}{m-1}\right]}\right\} \sum_{1 \leqslant i \leqslant I(s)}\left[r_{i}^{(s)}\right]^{n} .
$$

The first factor in the right-hand side of this inequality converges to zero as $s \rightarrow \infty$ while the second factor is bounded by (38). Thus by condition B2 and the strong convergence of $w_{s}$ to zero in $L_{m}(\Omega, N)$ it follows that the second term in (57) converges to zero as $s \rightarrow \infty$. Hence we obtain that $\lim _{s \rightarrow \infty} I_{42}^{(s)}=0$ and this shows that

$$
\lim _{s \rightarrow \infty} I_{4}^{(s)}=0
$$

since $I_{42}^{(s)}=0$. Relations (48), (49), (50), (52) and (58) imply that $w_{s}$ strongly converges to zero in $W_{m}^{1}(\Omega, N)$. The theorem is proved.

The results of this section establish the first claim in the main Theorem 3. The remaining of our work is devoted to the proof of the second claim of the theorem. We deal with this question in the next section.

\section{Derivation of the limit problem}

In this section we shall prove that the vector-function $u_{0}$, the weak limit of the sequence of solutions of problem (1)-(2) is a solution of problem (24)-(25). Let $g$ be an arbitrary vector-function in $\mathbf{C}_{o}^{\infty}(\Omega, N)$. We consider the sequence of functions

$$
g_{s}(x)=g(x)+\sum_{k=1}^{3} \rho_{s}^{(k)}(x)
$$

where

$$
\begin{gathered}
\rho_{s}^{(1)}(x)=\sum_{i=1}^{I(s)}\left[g_{i}^{(s)}-g(x)\right] \psi_{i}^{(s)}(x), \\
\rho_{s}^{(2)}(x)=-\sum_{\alpha=1}^{N} \sum_{i \in I_{s \alpha}^{\prime}} w_{i \alpha}^{(s)}(x) g_{i \alpha}^{(s)} \varphi_{i}^{(s)}(x), \\
\rho_{s}^{(3)}(x)=-\sum_{\alpha=1}^{N} \sum_{i \in I_{s \alpha}^{\prime \prime}} v_{i \alpha}^{(s)}(x, 1) g_{i \alpha}^{(s)} \varphi_{i}^{(s)}(x),
\end{gathered}
$$

where

$$
w_{i \alpha}^{(s)}(x)= \begin{cases}\frac{1}{\left(f_{i}^{(s)}-u_{i}^{(s)}\right)_{\alpha}} v_{i \alpha}^{(s)}\left(x, f_{i}^{(s)}-u_{i}^{(s)}\right), & \text { if }\left(f_{i}^{(s)}-u_{i}^{(s)}\right)_{\alpha} \neq 0, \\ 0, & \text { if }\left(f_{i}^{(s)}-u_{i}^{(s)}\right)_{\alpha}=0\end{cases}
$$


$(\cdot)_{\alpha}$ denotes the $\alpha$ th component of the vector $\cdot, g_{i}^{(s)}$ is the mean of the vector-function $g$ over the ball $B\left(x_{i}^{(s)}, \theta_{2} \rho_{i}^{(s)}\right), v_{i \alpha}^{(s)}\left(x, f_{i}^{(s)}-u_{i}^{(s)}\right)$ is a solution of problem (21)-(22) with $k=\left|\left(f_{i}^{(s)}-u_{i}^{(s)}\right)_{\alpha}\right|$ and $\vec{e}$ the $N$-dimensional vector whose $\alpha$ th component is $\left(f_{i}^{(s)}-u_{i}^{(s)}\right)_{\alpha} /\left|\left(f_{i}^{(s)}-u_{i}^{(s)}\right)_{\alpha}\right|$ and the remaining components are zero, $v_{i \alpha}^{(s)}(x, 1)$ is a solution of problem (21)-(22) with $k=1$ and the vector $\vec{e}$ has its $\alpha$ th component equal to 1 and the remaining components zero, $\psi_{i}^{(s)}$ and $\varphi_{i}^{(s)}$ are the test functions defined in the previous section. By the symbols $I_{s \alpha}^{\prime}$ and $I_{s \alpha}^{\prime \prime}$ we mean the sets

$$
\begin{aligned}
& I_{s \alpha}^{\prime}=\left\{i=1, \ldots, I(s):\left|\left(f_{i}^{(s)}-u_{i}^{(s)}\right)_{\alpha}\right| \geqslant d_{i}^{(s)}\right\}, \\
& I_{s \alpha}^{\prime \prime}=\left\{i=1, \ldots, I(s):\left|\left(f_{i}^{(s)}-u_{i}^{(s)}\right)_{\alpha}\right|<d_{i}^{(s)}\right\} .
\end{aligned}
$$

Using the same arguments as in the proof of Theorem 7, we can show that the sequences $\rho_{1}^{(s)}$ and $\rho_{3}^{(s)}$ strongly converge to zero in $W_{m}^{1}(\Omega, N)$, i.e.,

$$
\lim _{s \rightarrow \infty}\left\{\left\|\rho_{1}^{(s)}\right\|_{W_{m}^{1}(\Omega, N)}+\left\|\rho_{3}^{(s)}\right\|_{W_{m}^{1}(\Omega, N)}\right\}=0 .
$$

We also get that

$$
\left\|\rho_{2}^{(s)}\right\|_{W_{m}^{1}(\Omega, N)}<\infty
$$

and

$$
\lim _{s \rightarrow \infty}\left\|\rho_{2}^{(s)}\right\|_{W_{p}^{1}(\Omega, N)}=0
$$

for all $p \in(1, m)$.

A simple verification shows that $g_{s} \in \stackrel{\circ}{W_{m}^{1}}\left(\Omega^{(s)}, N\right)$. Hence we can substitute $\varphi(x)=$ $g_{s}(x)$ in the integral identity (6). By doing so, we get

$$
J_{1}^{(s)}+J_{2}^{(s)}+J_{3}^{(s)}+J_{4}^{(s)}=0,
$$

where

$$
\begin{gathered}
J_{1}^{(s)}=\sum_{j=1}^{N} \sum_{l=1}^{n} \int_{\Omega} A_{l}^{j}\left(x, \frac{\partial u_{s}}{\partial x}\right) \frac{\partial g_{j}}{\partial x_{l}} d x, \\
J_{2}^{(s)}=\sum_{j=1}^{N} \sum_{l=1}^{n} \int_{\Omega} A_{l}^{j}\left(x, \frac{\partial u_{s}}{\partial x}\right)\left(\frac{\partial \rho_{s j}^{(1)}}{\partial x_{l}}+\frac{\partial \rho_{s j}^{(3)}}{\partial x_{l}}\right) d x, \\
J_{3}^{(s)}=\sum_{j=1}^{N} \sum_{l=1}^{n} \int_{\Omega} A_{l}^{j}\left(x, \frac{\partial u_{s}}{\partial x}\right) \frac{\partial \rho_{s j}^{(2)}}{\partial x_{l}} d x .
\end{gathered}
$$

We investigate the behavior of each $J_{k}^{(s)}(k=1,2,3)$ as $s \rightarrow \infty$.

We have

$$
J_{1}^{(s)}=\sum_{j=1}^{N} \sum_{l=1}^{n} \int_{\Omega} A_{l}^{j}\left(x, \frac{\partial u_{0}}{\partial x}\right) \frac{\partial g_{j}}{\partial x_{l}} d x+J_{11}^{(s)},
$$


where

$$
J_{11}^{(s)}=\sum_{j=1}^{N} \sum_{l=1}^{n} \int_{\Omega}\left[A_{l}^{j}\left(x, \frac{\partial u_{s}}{\partial x}\right)-A_{l}^{j}\left(x, \frac{\partial u_{0}}{\partial x}\right)\right] \frac{\partial g_{j}}{\partial x_{l}} d x .
$$

From condition 2) and Hölder's inequality we have

$$
\begin{aligned}
J_{11}^{(s)} \leqslant & C^{\prime}\left\{\int_{\Omega}\left[\left|\frac{\partial u_{0}}{\partial x}\right|^{m}+\left|\frac{\partial u_{s}}{\partial x}\right|^{m}\right] d x\right\}^{\frac{m-2}{m}}\left\{\int_{\Omega}\left|\frac{\partial\left(u_{s}-u_{0}\right)}{\partial x}\right|^{m-1} d x\right\}^{\frac{1}{m-1}} \\
& +C^{\prime \prime}\left\{\int_{\Omega}\left|\frac{\partial\left(u_{s}-u_{0}\right)}{\partial x}\right|^{m-\varepsilon} d x\right\}^{\frac{1}{m-\varepsilon}},
\end{aligned}
$$

with $\varepsilon \in(0,1)$. Since $u_{s}$ strongly converges to $u_{0}$ in $W_{p}^{1}(\Omega, N)$ for any $p \in(1, m)$, and the first factor in the second inequality in (64) is bounded, we see that $J_{11}^{(s)}$ converges to zero as $s \rightarrow \infty$. Thus

$$
\lim _{s \rightarrow \infty} J_{1}^{(s)}=\sum_{j=1}^{N} \sum_{l=1}^{n} \int_{\Omega} A_{l}^{j}\left(x, \frac{\partial u_{0}}{\partial x}\right) \frac{\partial g_{j}}{\partial x_{l}} d x .
$$

Since $\rho_{s}^{(1)}$ and $\rho_{s}^{(3)}$ strongly converge to zero in $W_{m}^{1}(\Omega, N)$ then by condition 2$)$ and Hölder's inequality, it follows that

$$
\lim _{s \rightarrow \infty} J_{2}^{(s)}=0 .
$$

Using expansion (41), let us rewrite $J_{3}^{(s)}$ as follows

$$
\begin{aligned}
J_{3}^{(s)}=J_{31}^{(s)}+J_{32}^{(s)} \equiv & \sum_{j=1}^{N} \sum_{l=1}^{n} \int_{\Omega}\left[A_{l}^{j}\left(x, \frac{\partial u_{s}}{\partial x}\right)-A_{l}^{j}\left(x, \frac{\partial R_{s}^{(4)}}{\partial x}\right)\right] \frac{\partial \rho_{s j}^{(2)}}{\partial x_{l}} d x \\
& +\sum_{j=1}^{N} \sum_{l=1}^{n} \int_{\Omega} A_{l}^{j}\left(x, \frac{\partial R_{s}^{(4)}}{\partial x}\right) \frac{\partial \rho_{s j}^{(2)}}{\partial x_{l}} d x .
\end{aligned}
$$

The inequality (5) from condition 2) applied to $J_{31}^{(s)}$ yields

$$
\begin{aligned}
J_{31}^{(s)} \leqslant C\left\{J_{31}^{(s) 1}+J_{32}^{(s) 2}\right\} \equiv & C\left\{\int_{\Omega}\left|\frac{\partial \rho_{s}^{(2)}}{\partial x}\right|\left(\sum_{k=1}^{3}\left|\frac{\partial R_{s}^{(k)}}{\partial x}\right|+\left|\frac{\partial w_{s}}{\partial x}\right|\right)\right. \\
& \times\left(1+\left\lceil\left|\frac{\partial u_{s}}{\partial x}\right|+\left|\frac{\partial R_{s}^{(4)}}{\partial x}\right|\right]^{m-2}\right) d x \\
& \left.+\int_{\Omega}\left|\frac{\partial \rho_{s}^{(2)}}{\partial x}\right|\left|\frac{\partial u_{0}}{\partial x}\right|\left[1+\left|\frac{\partial R_{s}^{(4)}}{\partial x}\right|^{m-2}\right] d x\right\} .
\end{aligned}
$$

By Hölder's inequality, we have 


$$
\begin{aligned}
J_{31}^{(s) 1} \leqslant & \left\{\int_{\Omega}\left|\frac{\partial \rho_{s}^{(2)}}{\partial x}\right|^{m} d x\right\}^{\frac{1}{m}}\left\{\int_{\Omega}\left(\sum_{k=1}^{3}\left|\frac{\partial R_{s}^{(k)}}{\partial x}\right|+\left|\frac{\partial w_{s}}{\partial x}\right|\right)^{m} d x\right\}^{\frac{1}{m}} \\
& \times\left\{\int_{\Omega}\left(1+\left|\frac{\partial u_{s}}{\partial x}\right|+\left|\frac{\partial R_{s}^{(4)}}{\partial x}\right|\right)^{m} d x\right\}^{\frac{m-2}{m}} .
\end{aligned}
$$

The second factor in the right-hand side of this inequality converges to zero by Theorem 8 , while the other factors are bounded. Thus

$$
\lim _{s \rightarrow \infty} J_{31}^{(s) 1}=0 .
$$

By Hölder's inequality, we have

$$
J_{31}^{(s) 2} \leqslant\left\{\int_{\Omega}\left[1+\left|\frac{\partial u_{s}}{\partial x}\right|+\left|\frac{\partial R_{s}^{(4)}}{\partial x}\right|\right]^{m} d x\right\}^{\frac{m-2}{m}}\left\{\int_{\Omega}\left[\left|\frac{\partial \rho_{s}^{(2)}}{\partial x}\right|\left|\frac{\partial u_{0}}{\partial x}\right|\right]^{\frac{m}{2}} d x\right\}^{\frac{2}{m}} .
$$

Let us show that the second factor (that we denote by $H_{1}^{(s)}$ ) in this inequality converges to zero. Indeed applying Hölder's inequality, taking account of the properties of the functions $v_{i \alpha}^{(s)}$ and $\varphi_{i}^{(s)}$, and appealing to the estimate (27) in Theorem 5, we get

$$
\begin{aligned}
H_{1}^{(s)}= & \int_{\Omega}\left[\left|\frac{\partial \rho_{s}^{(2)}}{\partial x} \| \frac{\partial u_{0}}{\partial x}\right|\right]^{\frac{m}{2}} d x \\
\leqslant & C\left\{\sum_{i \in I_{s}^{\prime}}\left[d_{i}^{(s)}\right]^{n-m}\right\}^{\frac{1}{2}}\left\{\int_{\Omega}\left|\frac{\partial u_{0}}{\partial x}\right|^{m} d x\right\}^{\frac{1}{2}} \\
& +C\left\{\sum_{i \in I_{s}^{\prime \prime}}\left[d_{i}^{(s)}\right]^{n-m}\right\}^{\frac{1}{2}}\left\{\sum_{i \in I_{s}^{\prime \prime}} \int\left|\frac{\partial u_{0}}{\partial x}\right|^{m} d x\right\}^{\frac{1}{2}} .
\end{aligned}
$$

The first term in the right-hand side of this inequality converges to zero by (36), the second term also converges to zero by (39), (40) and the absolute continuity of an integral. Hence $H_{1}^{(s)}$ converges to zero as $s \rightarrow \infty$. Thus from (69), we get $\lim _{s \rightarrow \infty} J_{31}^{(s) 2}=0$. This together with (68) and (67) shows that $\lim _{s \rightarrow \infty} J_{31}^{(s)}=0$.

Now we are left with the investigation of $J_{32}^{(s)}$. Using the definition of $R_{s}^{(4)}$, we rewrite $J_{32}^{(s)}$ as follows.

$$
J_{32}^{(s)}=-\sum_{j=1}^{N} \sum_{l=1}^{n} \sum_{i=1}^{I(s)} g_{i \alpha}^{(s)} \int_{\Omega} A_{l}^{j}\left(x, \frac{\partial v_{i}^{(s)}}{\partial x}\right) \frac{\partial w_{i \alpha, j}^{(s)}}{\partial x_{l}} d x+J_{32}^{(s) 1}+J_{32}^{(s) 2},
$$

where

$$
J_{32}^{(s) 1}=\sum_{\alpha, j=1}^{N} \sum_{l=1}^{n} \sum_{i \notin I_{s}^{\prime \prime} \cap I_{s \alpha}^{\prime}} g_{i \alpha}^{(s)} \int_{\Omega} A_{l}^{j}\left(x, \frac{\partial v_{i}^{(s)}}{\partial x}\right) \frac{\partial w_{i \alpha, j}^{(s)}}{\partial x} d x
$$




$$
\begin{aligned}
J_{32}^{(s) 2}= & \sum_{\alpha, j=1}^{N} \sum_{l=1}^{n} \sum_{i \in I_{s}^{\prime \prime} \cap I_{s \alpha}^{\prime}} g_{i \alpha}^{(s)} \int_{\Omega}\left\{A_{l}^{j}\left(x, \frac{\partial\left[v_{i}^{(s)} \varphi_{i}^{(s)}\right]}{\partial x}\right) \frac{\partial\left[\varphi_{i}^{(s)} w_{i \alpha, j}^{(s)}\right]}{\partial x_{l}}\right. \\
& \left.-A_{l}^{j}\left(x, \frac{\partial v_{i}^{(s)}}{\partial x}\right) \frac{\partial w_{i \alpha, j}^{(s)}}{\partial x_{l}}\right\} d x,
\end{aligned}
$$

where $w_{i \alpha, j}^{(s)}$ is the $j$ th component of $w_{i \alpha}^{(s)}$. By condition 2) and Hölder's inequality, we have

$$
J_{32}^{(s) 1} \leqslant C\left\{H_{31}^{(s)} \times H_{32}^{(s)}\right\}
$$

where

$$
H_{31}^{(s)}=\sum_{i \notin I_{s}^{\prime \prime} \cap I_{s \alpha}^{\prime}}\left\{\int_{D_{i}^{(s)}}\left(1+\left|\frac{\partial v_{i}^{(s)}}{\partial x}\right|\right)^{m} d x\right\}^{\frac{m-2}{m}}\left\{\int_{D_{i}^{(s)}}\left|\frac{\partial v_{i}^{(s)}}{\partial x}\right|^{m} d x\right\}^{\frac{1}{m}}
$$

and

$$
H_{32}^{(s)}=\sum_{\alpha=1}^{N} \sum_{i \notin I_{s}^{\prime \prime} \cap I_{s \alpha}^{\prime}}\left\{\int_{D_{i}^{(s)}}\left|\frac{\partial w_{i \alpha}^{(s)}}{\partial x}\right|^{m}\right\}^{\frac{1}{m}}
$$

Noticing that $\{i: i=1, \ldots, I(s)\} \backslash\left[I_{s}^{\prime \prime} \cap I_{s \alpha}^{\prime}\right]=I_{s}^{\prime} \cup\left[I_{s}^{\prime \prime} \cap I_{s \alpha}^{\prime \prime}\right]$, by Theorem 5, we get

$$
\begin{aligned}
H_{31}^{(s)} & \leqslant C \sum_{\alpha=1}^{N} \sum_{i \notin I_{s}^{\prime \prime} \cap I_{s \alpha}^{\prime}}\left[d_{i}^{(s)}\right]^{n-m}\left|\left(f_{i}^{(s)}-u_{i}^{(s)}\right)_{\alpha}\right|^{m} \\
& \leqslant C\left\{\sum_{i \in I_{s}^{\prime}}\left[d_{i}^{(s)}\right]^{n-m}+\sum_{i \in I_{s}^{\prime \prime} \cap I_{s \alpha}^{\prime \prime}}\left[\rho_{i}^{(s)}\right]^{n}\right\} .
\end{aligned}
$$

Here we have used the definition of the sets $I_{s}^{\prime \prime}$ and $I_{s \alpha}^{\prime \prime}$. By (36) and (37), we conclude from this inequality that $\lim _{s \rightarrow \infty} H_{31}^{(s)}=0$. Analogous arguments show that $\lim _{s \rightarrow \infty} H_{32}^{(s)}=0$. Thus

$$
\lim _{s \rightarrow \infty} J_{32}^{(s) 1}=0
$$

Next, we write

$$
J_{32}^{(s) 2}=L_{1}^{(s)}+L_{2}^{(s)}+L_{3}^{(s)}
$$

where

$$
\begin{aligned}
L_{1}^{(s)}= & -\sum_{\alpha, j=1}^{N} \sum_{l=1}^{n} \sum_{i \in I_{s}^{\prime \prime} \cap I_{s \alpha}^{\prime}} g_{i \alpha}^{(s)} \int_{\Omega}\left\{A_{l}^{j}\left(x, \frac{\partial\left[v_{i}^{(s)} \varphi_{i}^{(s)}\right]}{\partial x}\right) \frac{\partial\left[\varphi_{i}^{(s)} w_{i \alpha, j}^{(s)}\right]}{\partial x_{l}}\right. \\
& \left.-A_{l}^{j}\left(x, \frac{\partial v_{i}^{(s)}}{\partial x}\right) \varphi_{i}^{(s)} \frac{\partial w_{i \alpha, j}^{(s)}}{\partial x_{l}}\right\} d x \\
L_{2}^{(s)}= & \sum_{\alpha, j=1}^{N} \sum_{l=1}^{n} \sum_{i \in I_{s}^{\prime \prime} \cap I_{s \alpha}^{\prime}} g_{i \alpha}^{(s)} \int_{\Omega}\left(1-\varphi_{i}^{(s)}\right) A_{l}^{j}\left(x, \frac{\partial v_{i}^{(s)}}{\partial x}\right) \frac{\partial w_{i \alpha, j}^{(s)}}{\partial x_{l}} d x
\end{aligned}
$$




$$
L_{3}^{(s)}=-\sum_{\alpha, j=1}^{N} \sum_{l=1}^{n} \sum_{i \in I_{s}^{\prime \prime} \cap I_{s \alpha}^{\prime}} g_{i \alpha}^{(s)} \int_{\Omega} w_{i \alpha, j}^{(s)} A_{l}^{j}\left(x, \frac{\partial\left[v_{i}^{(s)} \varphi_{i}^{(s)}\right]}{\partial x}\right) \frac{\partial \varphi_{i}^{(s)}}{\partial x_{l}} d x .
$$

From condition 2), we have

$$
L_{1}^{(s)} \leqslant C\left\{L_{11}^{(s)}+L_{12}^{(s)}\right\}
$$

where

$$
\begin{aligned}
L_{11}^{(s)}= & \sum_{\alpha=1}^{N} \sum_{i \in I_{s}^{\prime \prime} \cap I_{s \alpha}^{\prime}}\left|g_{i \alpha}^{(s)}\right| \int_{\Omega}\left[\left|\frac{\partial\left[\varphi_{i}^{(s)} v_{i}^{(s)}\right]}{\partial x}\right|+\left|\frac{\partial v_{i}^{(s)}}{\partial x}\right|\right]^{m-2} \\
& \times\left|\frac{\partial\left[\left(1-\varphi_{i}^{(s)}\right) v_{i}^{(s)}\right]}{\partial x}\right|\left|\frac{\partial w_{i \alpha}^{(s)}}{\partial x}\right| d x
\end{aligned}
$$

and

$$
L_{12}^{(s)}=\sum_{\alpha=1}^{N} \sum_{i \in I_{s}^{\prime \prime} \cap I_{s \alpha}^{\prime}}\left|g_{i \alpha}^{(s)}\right| \int_{\Omega}\left|\frac{\partial\left[\left(1-\varphi_{i}^{(s)}\right) v_{i}^{(s)}\right]}{\partial x}\right|\left|\frac{\partial w_{i \alpha}^{(s)}}{\partial x}\right| d x .
$$

Let us estimate $L_{11}^{(s)}$. Denote $B\left(x_{i}^{(s)}, \theta_{3} \rho_{i}^{(s)}\right)$ by $E_{i}^{(s)}$. From the definition of $\varphi_{i}^{(s)}$ and Young's inequality, we have

$$
L_{11}^{(s)} \leqslant C \sum_{\alpha=1}^{N} \sum_{i \in I_{s}^{\prime \prime} \cap I_{s \alpha}^{\prime}} \int_{D_{i}^{(s)} \backslash E_{i}^{(s)}}\left\{\left|\frac{\partial v_{i}^{(s)}}{\partial x}\right|^{m}+\left|v_{i}^{(s)}\right|^{m}\left|\frac{\partial \varphi_{i}^{(s)}}{\partial x}\right|^{m}+\left|\frac{\partial w_{i \alpha}^{(s)}}{\partial x}\right|^{m}\right\} d x .
$$

Let

$$
\begin{array}{ll}
m_{i}^{(s)}=\max _{x \in D_{i}^{(s)} \backslash E_{i}^{(s)}}\left|v_{i}^{(s)}\left(x, f_{i}^{(s)}-u_{i}^{(s)}\right)\right|, & m_{i \alpha}^{(s)}=\max _{x \in D_{i}^{(s)} \backslash E_{i}^{(s)}}\left|v_{i \alpha}^{(s)}\left(x, f_{i}^{(s)}-u_{i}^{(s)}\right)\right|, \\
E_{m_{i}^{(s)}}=\left\{x \in D_{i}^{(s)}:\left|v_{i}^{(s)}\right| \leqslant m_{i}^{(s)}\right\}, & E_{m_{i \alpha}^{(s)}}=\left\{x \in D_{i}^{(s)}:\left|v_{i \alpha}^{(s)}\right| \leqslant m_{i \alpha}^{(s)}\right\} .
\end{array}
$$

By Theorem 5 and the definition of the set $I_{s}^{\prime \prime}$, we get

$$
\begin{aligned}
L_{11}^{(s)} & \leqslant C \sum_{\alpha=1}^{N} \sum_{i \in I_{s}^{\prime \prime} \cap I_{s \alpha}^{\prime}}\left\{\left[m_{i}^{(s)}\right]^{m}\left[\rho_{i}^{(s)}\right]^{n-m}+\int_{E_{m_{i}^{(s)}}}\left|\frac{\partial v_{i}^{(s)}}{\partial x}\right|^{m} d x+\int_{E_{m_{i \alpha}^{(s)}}}\left|\frac{\partial v_{i \alpha}^{(s)}}{\partial x}\right|^{m} d x\right\} \\
& \leqslant C \max _{1 \leqslant i \leqslant I(s)} \frac{1}{\ln ^{2 \frac{n-m}{m-1}} r_{i}^{(s)}} \sum_{i=1}^{I(s)}\left[\frac{\left[d_{i}^{(s)}\right]^{m(n-m)}}{\left[r_{i}^{(s)}\right]^{n}}\right]^{\frac{1}{m-1}} .
\end{aligned}
$$

A passage to the limit in this inequality and condition B2 yield that

$$
\lim _{s \rightarrow \infty} L_{11}^{(s)}=0
$$


For the estimation of $L_{12}^{(s)}$ we use Hölder's and Young's inequalities to get

$$
L_{12}^{(s)} \leqslant C\left\{\sum_{\alpha=1}^{N} \sum_{i \in I_{s}^{\prime \prime} \cap I_{s \alpha}^{\prime}} \int_{D_{i}^{(s)} \backslash E_{i}^{(s)}}\left(\left|\frac{\partial\left[\left(1-\varphi_{i}^{(s)}\right) v_{i}^{(s)}\right]}{\partial x}\right|^{m}+\left|\frac{\partial w_{i \alpha}^{(s)}}{\partial x}\right|^{m}\right) d x\right\}^{\frac{2}{m}} .
$$

Arguing as in the estimation of $L_{11}^{(s)}$, we see that $\lim _{s \rightarrow \infty} L_{12}^{(s)}=0$. This fact together with (74) and (73) imply that

$$
\lim _{s \rightarrow \infty} L_{1}^{(s)}=0 .
$$

The convergence of $L_{2}^{(s)}$ and $L_{3}^{(s)}$ to zero as $s \rightarrow \infty$ is established analogously. Hence we have shown that

$$
\lim _{s \rightarrow \infty} J_{32}^{(s) 2}=0 .
$$

We still have to investigate the first term in the right-hand side of (70). In particular we shall show that

$$
\begin{aligned}
& \lim _{s \rightarrow \infty} \sum_{\alpha, j=1}^{N} \sum_{l=1}^{n} \sum_{i=1}^{I(s)} g_{i \alpha}^{(s)} \int_{\Omega} A_{l}^{j}\left(x, \frac{\partial v_{i}^{(s)}}{\partial x}\right) \frac{\partial w_{i \alpha, j}^{(s)}}{\partial x_{l}} d x \\
& =\sum_{\alpha, j=1}^{N} \int_{\Omega} g_{\alpha}(x) C^{(j)}\left(x, f-u_{0}\right) \delta_{\alpha}^{j} d x,
\end{aligned}
$$

where $\delta_{\alpha}^{j}=1$ if $\alpha=j$, and $\delta_{\alpha}^{j}=0$ if $\alpha \neq j$; the function $C^{(j)}\left(x, f-u_{0}\right)$ is defined as in (23).

Since $u_{0} \in W_{m}^{1}(\Omega, N)$, there exists a uniformly bounded sequence of infinitely differentiable functions $u_{k}(x), k=1,2, \ldots$, which converges to $u_{0}$ in $W_{m}^{1}(\Omega, N)$ as $s \rightarrow \infty$. For a given $k$ let there be defined a number $d=d(k)>0$ such that for any set $E \subset \Omega$ with its diameter less than $2 d$,

$$
\max _{x, y}\left\{\left|u_{k}(x)-u_{k}(y)\right|+|f(x)-f(y)|+|g(x)-g(y)|\right\}<k^{-(m-1)} .
$$

Let us represent $\bar{\Omega}=\bigcup_{t} \Omega_{t}, t=1, \ldots, T(k), \Omega_{t} \cap \Omega_{t^{\prime}}=\emptyset$ for $t \neq t^{\prime}$, where the $\Omega_{t}$ are some sets with piecewise smooth boundaries such that their diameters is less than $d$ for all $t$. Let $s_{1}=s_{1}(k)$ be such that for $s \geqslant s_{1}(k)$ and $i=1, \ldots, I(s)$, the inequality $d_{i}^{(s)}+r_{i}^{(s)}<d$ holds. Let $I_{s}\left(\Omega_{t}\right)=\left\{i \in\{1, \ldots, I(s)\}: x_{i}^{(s)} \in \Omega_{t}\right\}$. We have the following representation

$$
\begin{aligned}
& \sum_{\alpha, j=1}^{N} \sum_{l=1}^{n} \sum_{i=1}^{I(s)} g_{i \alpha}^{(s)} \int_{\Omega} A_{l}^{j}\left(x, \frac{\partial v_{i}^{(s)}}{\partial x}\right) \frac{\partial w_{i \alpha, j}^{(s)}}{\partial x_{l}} d x \\
& \quad=\sum_{t=1}^{T} \sum_{\alpha, j=1}^{N} \sum_{l=1}^{n} \sum_{i \in I_{s}\left(\Omega_{t}\right)} g_{i \alpha}^{(s)} \int_{\Omega_{t}} A_{l}^{j}\left(x, \frac{\partial v_{i}^{(s)}}{\partial x}\right) \frac{\partial w_{i \alpha, j}^{(s)}}{\partial x_{l}} d x \\
& =M_{1}^{(s)}+M_{2}^{(s)}+M_{3}^{(s)},
\end{aligned}
$$


where

$$
\begin{aligned}
M_{1}^{(s)}= & \sum_{t=1}^{T} \sum_{\alpha, j=1}^{N} \sum_{i \in I_{s}\left(\Omega_{t}\right)} g_{i \alpha}^{(s)}\left\{\sum_{l=1}^{n} \int_{\Omega_{t}} A_{l}^{j}\left(x, \frac{\partial v_{i}^{(s)}}{\partial x}\right) \frac{\partial w_{i \alpha, j}^{(s)}}{\partial x_{l}} d x\right. \\
& \left.-g_{i \alpha}^{(s)} \int_{\Omega_{t}} C^{(j)}\left(x, f_{i}^{(s)}-u_{i}^{(s)}\right) \delta_{\alpha}^{j} d x\right\}, \\
M_{2}^{(s)}= & \sum_{t=1}^{T} \sum_{\alpha, j=1}^{N} \sum_{i \in I_{s}\left(\Omega_{t}\right)}\left\{g_{i \alpha}^{(s)} \int_{\Omega_{t}} C^{(j)}\left(x, f_{i}^{(s)}-u_{i}^{(s)}\right) \delta_{\alpha}^{j} d x\right. \\
& \left.-\int_{\Omega_{t}} g_{\alpha}(x) C^{(j)}\left(x, f-u_{0}\right) \delta_{\alpha}^{j} d x\right\}, \\
M_{3}^{(s)}= & \sum_{j, \alpha=1}^{N} \int_{\Omega} g_{\alpha}(x) C^{(j)}\left(x, f_{i}^{(s)}-u_{i}^{(s)}\right) \delta_{\alpha}^{j} d x .
\end{aligned}
$$

From the assumption C, we see that for any $\Omega_{t}$ in the partition of $\Omega$ and $s$ sufficiently large

$$
\sum_{j=1}^{N} \sum_{i \in I_{s}\left(\Omega_{t}\right)}\left[\int_{\Omega_{t}} A_{l}^{j}\left(x, \frac{\partial v_{i}^{(s)}}{\partial x}\right) \frac{\partial w_{i \alpha, j}^{(s)}}{\partial x} d x-\int_{\Omega_{t}} C^{(\alpha)}\left(x, f_{i}^{(s)}-u_{i}^{(s)}\right) d x\right] \leqslant \alpha_{1 s},
$$

with $\lim _{s \rightarrow \infty} \alpha_{1 s}=0$. This implies that

$$
\lim _{s \rightarrow \infty} M_{11}^{(s)}=0 .
$$

From the definition of $C^{(j)}(x, \cdot)$ and Theorem 6 , we have for $\bar{k}=\left(k_{1}, \ldots, k_{N}\right)$ and $\hat{k}=\left(\hat{k}_{1}, \ldots, \hat{k}_{N}\right)$,

$$
\left|C^{(j)}(x, \bar{k})-C^{(j)}(x, \hat{k})\right| \leqslant C|\bar{k}-\hat{k}|^{\frac{1}{m-1}}\left[d_{i}^{(s)}\right]^{n-m},
$$

for $|\bar{k}|,|\hat{k}|<\infty$. This inequality together with (77) and Theorem 5 imply

$$
\begin{aligned}
M_{2}^{(s)}= & \sum_{t=1}^{T} \sum_{\alpha, j=1}^{N} \sum_{i \in I_{s}\left(\Omega_{t}\right)}\left\{\int_{\Omega_{t}}\left(g_{i \alpha}^{(s)}-g_{\alpha}(x)\right) C^{(j)}\left(x, f_{i}^{(s)}-u_{i}^{(s)}\right) \delta_{\alpha}^{j} d x\right. \\
& \left.-\int_{\Omega_{t}} g_{\alpha}(x)\left(C^{(j)}\left(x, f_{i}^{(s)}-u_{i}^{(s)}\right)-C^{(j)}\left(x, f-u_{0}\right)\right) \delta_{\alpha}^{j} d x\right\} \\
\leqslant & C \sum_{t=1}^{T} \sum_{\alpha, j=1}^{N} \sum_{i \in I_{s}\left(\Omega_{t}\right)}\left\{\left|g_{i \alpha}^{(s)}-g_{\alpha}(x)\right|+\left|f_{i}^{(s)}-f(x)\right|^{\frac{1}{m-1}}\right. \\
& \left.+\left|u_{i}^{(s)}-u_{0}(x)\right|^{\frac{1}{m-1}}\right\} \times\left[d_{i}^{(s)}\right]^{n-m}
\end{aligned}
$$




$$
\leqslant \frac{C}{k} \sum_{i=1}^{I(s)}\left[d_{i}^{(s)}\right]^{n-m}=\alpha_{k}
$$

By (40), it is clear that $\lim _{k \rightarrow \infty} \alpha_{k}=0$.

Recapitulating all that has been done so far in this section, we conclude that

$$
\sum_{j, \alpha=1}^{N} \int_{\Omega}\left\{\sum_{l=1}^{n} A_{l}^{j}\left(x, \frac{\partial u_{0}}{\partial x}\right) \frac{\partial g_{j}}{\partial x_{l}}+g_{\alpha}(x) C^{(j)}\left(x, f(x)-u_{0}(x)\right) \delta_{\alpha}^{j}\right\} d x=\beta_{s}+\alpha_{k}
$$

where $\alpha_{k}$ is from (80) and $\beta_{s} \rightarrow 0$, as $s \rightarrow \infty$. Since the left-hand side in this equation is independent of $k$ and $s$, then we can make $\alpha_{k}$ and $\beta_{s}$ as small as we wish for $k$ and $s$, respectively large. This implies that $u_{0}$ satisfies the integral identity

$$
\sum_{j, \alpha=1}^{N} \int_{\Omega}\left\{\sum_{l=1}^{n} A_{l}^{j}\left(x, \frac{\partial u_{0}}{\partial x}\right) \frac{\partial g_{j}}{\partial x_{l}}+g_{\alpha}(x) C^{(j)}\left(x, f(x)-u_{0}(x)\right) \delta_{\alpha}^{j}\right\} d x=0,
$$

for all $g \in \mathbf{C}_{o}^{\infty}(\Omega, N)$. This completes the proof of Theorem 3 .

\section{Acknowledgements}

The author gratefully acknowledges the support of the National Research Foundation of South Africa through its Institutional Research Development Programme for HBUs; Grant GUN:2044642.

\section{REFERENCES}

[1] A. Bensoussan, J.L. Lions, G. Papanicolaou, Asymptotic Analysis for Periodic Structures, North-Holland, Amsterdam, 1978.

[2] J. Casado Diaz, Homogenization of Dirichlet problems for monotone operators in varying domains, Proc. Roy. Soc. Edinburgh A 127 (1997) 457-478.

[3] D. Cioranescu, F. Murat, Un terme etrange venu d'ailleurs I and II, in: Nonlinear PDE's and their Applications, in: Pitman Research Notes in Mathematics, Vol. 60, 1982, pp. 98-138; Vol. 70, 1983, pp. 154-178.

[4] G. Dal Maso, An Introduction to $\Gamma$-convergence, Birkhauser, Boston, 1993.

[5] G. Dal Maso, A. Garroni, New results on the asymptotic behavior of Dirichlet problems in perforated domains, Math. Mod. Meth. Appl. Sci. 3 (1994) 373-407.

[6] G. Dal Maso, F. Murat, Dirichlet problems in perforated domains for homogeneous operators on $H_{0}^{1}$, in: Calculus of Variations, Homogenization and Continuum Mechanics, World Scientific, Singapore, 1994, pp. 177-202.

[7] G. Dal Maso, F. Murat, Asymptotic behaviour and correctors for Dirichlet problems in perforated domains with homogeneous monotone operators, Ann. Scuola Norm. Sup. Pisa, Cl. Sci. 4 (1997) 24, No. 2, 239-290.

[8] O.A. Ladyzhenskaya, N.N. Uraltseva, Linear and Quasilinear Elliptic Equations, Academic Press, New-York, 1968.

[9] J. Leray, J.L. Lions, Quelques resultats de Viŝik sur les problèmes élliptiques nonlinéaires par la méthode de Minty-Browder, Bull. Soc. Math. France 93 (1965) 97-107. 
[10] V.A. Marchenko, E.Ya. Khruslov, Boundary Value Problems in Domains With Fine-Grained Boundaries, Naukova Dumka, Kiev, 1974 (Russian).

[11] J. Moser, A new proof of de Giorgi's theorem concerning the regularity problem for elliptic differential equations, Comm. Pure Appl. Math. 13 (1960) 457-468.

[12] O.A. Oleinik, A.S. Shamaev, G.A. Yocifian, Mathematical Problems in Elasticity and Homogenization, North-Holland, Amsterdam, 1992.

[13] M. Sango, Pointwise a priori estimates for solutions of a system of quasilinear elliptic equations, Applicable Analysis, to appear.

[14] M. Sango, Homogenization of the Dirichlet problem for a system of quasilinear elliptic equations in a perforated domain, C. R. Acad. Sci. Paris 329 (1999) 293-298.

[15] J. Serrin, Local behavior of solutions of quasi-linear equations, Acta Math. 111 (1964) 247302.

[16] I.V. Skrypnik, Quasilinear Dirichlet problem for domains with fine-grained boundary, Dokl. Akad. Nauk Ukrain. SSR Ser. A 2 (1982) 21-25.

[17] I.V. Skrypnik, Methods for Analysis of Nonlinear Elliptic Boundary Value Problems, Nauka, Moscow, 1990. English translation in: Translations of Mathematical Monographs, Vol. 139, AMS, Providence, 1994.

[18] I.V. Skrypnik, Homogenization of nonlinear Dirichlet problems in perforated domains of general type, Sbornik Mathematics 187 (1996) 1229-1260.

[19] V.V. Zhikov, S.M. Kozlov, O.A. Oleinik, Homogenization of Differential Operators and Integral Functionals, Springer-Verlag, Berlin, 1994. 